\title{
Phase-field modelling of brittle fracture in thin shell elements based on the MITC4+ approach
}

\author{
Udit Pillai · Savvas Triantafyllou - Ian Ashcroft • Yasser Essa • Federico \\ Martin de la Escalera
}

Received: date / Accepted: date

\begin{abstract}
We present a phase field based MITC4+ 28 shell element formulation to simulate fracture propa- 29 gation in thin shell structures. The employed MITC4+ 30 approach renders the element shear- and membrane- 31 locking free, hence providing high-fidelity fracture sim- 32 ulations in planar and curved topologies. To capture the 33 mechanical response under bending-dominated frac- 34 ture, a crack-driving force description based on the 35 maximum strain energy density through the shell- 36 thickness is considered. Several numerical examples 37 simulating fracture in flat and curved shell structures 38 are presented, and the accuracy of the proposed formu- 39 lation is examined by comparing the predicted critical $_{40}$ fracture loads against analytical estimates.

Keywords Mindlin shell elements . Shear and membrane locking - MITC4+ formulations · Phasefield implementation · Brittle fracture

\section{Introduction}

Thin shell structures find numerous applications in a ${ }^{49}$ wide range of industries within the aerospace, auto- 50 motive, and construction sectors. Thin composite lami- ${ }^{51}$ nates in particular are being deployed in aircraft struc- 52 tures and comprise the chassis of automotive vehicles. ${ }^{53}$ Hence, high-fidelity simulation of damage processes per- ${ }^{54}$ tinent to thin-shells is vital for estimating their critical ${ }^{55}$ load bearing capacities while at the same time reducing ${ }^{56}$ the number of high-cost experimental test.
\end{abstract}

Savvas Triantafyllou

Institute for Structural Engineering and Aseismic research,

School of Civil Engineering, National Technical University of ${ }^{60}$ Athens,

Zografou Campus, 15780, Athens, Greece

E-mail: savtri@mail.ntua.gr
Numerical simulation of evolving damage in thin shell-like structures is often performed using ReissnerMindlin shell elements which allow efficient modelling of both in-plane (membrane) and out-of-plane (bending) deformations at a reduced computational cost. Especially when using an explicit time-integration scheme, shell elements do not penalize the stable time-increment even when the thickness is extremely small [60]. This makes Mindlin shells an ideal candidate for modelling computationally complex fracture problems involving, e.g., impact driven damage scenarios.

Damage modelling methods can be broadly categorized onto two types, i.e., Discrete or Smeared/Diffuse. In discrete methods, a crack is treated either explicitly as a geometrical entity or implicitly as a discontinuity in the displacement field. In diffuse methods, the crack is smeared over the surrounding domain and the stress degradation effects are incorporated by means of a damage variable embedded directly into the constitutive formulations.

Discrete crack approaches primarily rely on modifying an existing finite element mesh in the locations where crack propagates, see, e.g., the robust remeshing algorithms developed by Ingraffea and Saouma [35], Bouchard et al [18, 19], Rethore et al [52], Shahani and Fasakhodi [57]. The extended finite element method (XFEM), first introduced in Belytschko and Black [13] [, see, also, 24], eliminates the need of expensive mesh-updating algorithms for tracking crack paths by decoupling the crack topology from the underlying finite-element mesh. The XFEM models cracks by introducing a set of additional (enriched) degrees of freedom and corresponding discontinuous basis functions. Over the past fifteen years, the method has evolved onto the industrial standard for resolving crack-tip stress singularities without the requirement of very fine discretiza- 
tions. However, the XFEM is not free from computa-116 tional complexities pertinent to the the number of ad-117 ditional DOFs; furthermore, it relies on the definition ${ }_{118}$ of ad-hoc assumptions vis-a-vis the stress field at the $\mathrm{e}_{119}$ crack-tip. Furthermore, the extension of XFEM to $3-\mathrm{D}_{120}$ problems is not straightforward and poses challenges $\mathrm{in}_{121}$ specifying the crack propagation increment in $3-\mathrm{D}[27]_{122}$

Cohesive Zone Modelling (CZM) is a discrete ${ }^{123}$ method $[25,10,34]$ that simulates fracture propaga- ${ }^{124}$ tion by redistributing the stresses ahead of the crack-tip ${ }^{125}$ over a finite fracture process zone (FPZ). The consti- ${ }^{126}$ tutive behaviour of the FPZ is defined on the basis of ${ }^{127}$ a traction-separation law. With the exception of the ${ }^{128}$ Cohesive Segments Method (CSM) [51], CZM relies on ${ }^{129}$ the pre-definition of the crack surfaces. Hence, it can- ${ }^{130}$ not predict arbitrary crack propagation scenarios and ${ }^{131}$ is mostly applied in cases where crack path is known ${ }^{132}$ a-priori, e.g., in composite delamination.

Diffuse damage modelling approaches such as the Phase-field method (PFM) [29, 20] and the thick level ${ }_{136}$ set method [43], overcome these challenges and have ${ }_{137}$ been proven robust in treating complex crack patterns, ${ }_{138}$ e.g., branching, merging, and curvilinear crack paths. The PFM emerged from the step-changing works of Francfort and Marigo [29], Bourdin et al [20] and has ${ }_{141}$ garnered much attention in the past 10 years. The main ${ }_{142}$ advantage of the PFM is that the crack initiation loca- ${ }_{143}$ tion and crack-paths do not need to be predefined, but ${ }_{144}$ naturally emerge from the solution of a PDE that is derived on the basis of energy-minimisation principles ${ }_{146}$ and solved over the entire computational domain. $\mathrm{The}_{147}$ PFM relies on replacing the sharp crack edges with $a_{148}$ diffusive crack interface represented by the phase field ${ }_{149}$ and hence resolves difficulties of numerically tracking discontinuities in the displacement field during crack $_{151}$ propagation. To this point, the PFM has been extended ${ }_{152}$ to treat brittle fracture $[41,40,44]$, ductile fracture ${ }_{153}$ $[4,17]$, hydraulic fracture $[62,33,28,47]$, and has also $_{154}$ been applied within material-point method (MPM) [37 $]_{155}$ and virtual-element method (VEM) setting [1].

Despite the significant advantages provided by shell157 elements in resolving three dimensional surfaces in $\mathrm{a}^{158}$ robust and efficient manner, there have been only lim-159 ited efforts to apply the PFM for simulating shell160 damage problems; a detailed review is provided in ${ }^{161}$ [63]. The PFM has been used to modelling thin-162 shell fractures based on the Kirchoff-Love shell the-163 ory $[7,61,38]$. Kiendl et al [38] adopted higher order ${ }^{164}$ smooth basis functions (NURBS), whereas Amiri et al165 [7] employed maximum entropy meshfree approxima-166 tions based on $C^{1}$ continuous basis functions. ReinosO167 et al [50] extended the PFM for brittle fracture in large-168 deformation solid shell elements based on enhanced assumed strain (EAS) formulations.

An important challenge to address when using thin Mindlin shell elements is that they display membrane and transverse shear locking [39], which significantly affects the evolution the simulated crack path. Transverse shear locking occurs purely due to the displacementbased interpolation that is also used for the calculation of strains. This leads to a significant over-prediction of the bending stiffness and an under-prediction of the transverse deformations which may become lower than the theoretical estimates by orders of magnitude [26]. In addition, when the shell elements are curved or become overly distorted during nonlinear deformation, spurious coupling may occur between membrane and transverse shear strains; this also increases the element stiffness and leads to membrane locking [39]. Since in thin shells the membrane stiffness can be significantly larger than the bending stiffness, membrane locking leads to the exclusion of the desired bending modes from the overall element response [23].

To this point, several approaches have been proposed to alleviate locking in shell elements. Selective/reduced integration schemes have been employed $[15,14,64]$, that however result in spurious zero energy modes necessitating additional hourglass stabilization techniques. More notably, the precise prediction of crack paths using elements based on reduced integration necessitates an even finer mesh discretisation in the critical regions which adds up to the computational complexity. The assumed strain approach based on the Mixed Interpolation of Tensorial Components (MITC) formulation proposed in the works of Dvorkin and Bathe [26], Bathe and Dvorkin [12], Bathe [11], and more recently the MITC4+ approach proposed by Ko et al [39] has been successful in alleviating both transverse shear and membrane locking issues and also pass all basic patch tests in an optimal convergence behaviour for both uniform and distorted meshes.

In this work, we extend the phase-field modelling framework to simulate brittle fracture in MITC4+ based thin Mindlin shell elements, wherein damage initiates and evolves due to coupled membrane/bending deformations. We restrict our implementation to thin 4-noded shell elements subjected to small strain deformations; however, the approach is general and can be straight-forwardly extended to higher order shell elements. We use the proposed formulation to examine the post-fracture response of 3D surfaces and establish its accuracy by comparing against analytically predicted critical fracture loads.

The paper is structured as follows: In Sec. 2, the geometrical and kinematic considerations for the Mindlin 


\subsection{Kinematics}

The displacement at any point $P$ lying above or below ${ }_{205}$ the shell mid-surface (Fig. 1a) is derived with respect ${ }_{206}$ to the mid-surface according to Eq. (2) [23].

$\mathbf{u}_{P}=\left[\begin{array}{c}u \\ v \\ w\end{array}\right]=\sum N_{i}\left(\left[\begin{array}{c}u_{i} \\ v_{i} \\ w_{i}\end{array}\right]+\zeta \frac{t_{i}}{2}\left[\mu_{i}\right]\left[\begin{array}{c}\alpha_{i} \\ \beta_{i} \\ \gamma_{i}\end{array}\right]\right)$

where $\mu_{i}$ contains the direction cosines of the shell vec- ${ }^{211}$ tors $\mathbf{V}_{1 i}$ and $\mathbf{V}_{2 i}$ and assumes the following form (Eq. ${ }_{213}$ (3))

$\left[\mu_{i}\right]=\left[-\frac{\mathbf{V}_{2 i}}{\left|\mathbf{V}_{2 i}\right|}, \frac{\mathbf{V}_{1 i}}{\left|\mathbf{V}_{1 i}\right|}, \mathbf{0}\right]=\left[\begin{array}{ccc}-l_{2 i} & l_{1 i} & 0 \\ -m_{2 i} & m_{1 i} & 0 \\ -n_{2 i} & n_{1 i} & 0\end{array}\right]$. 217

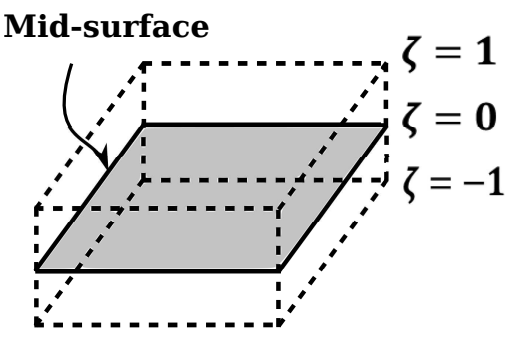

(a)

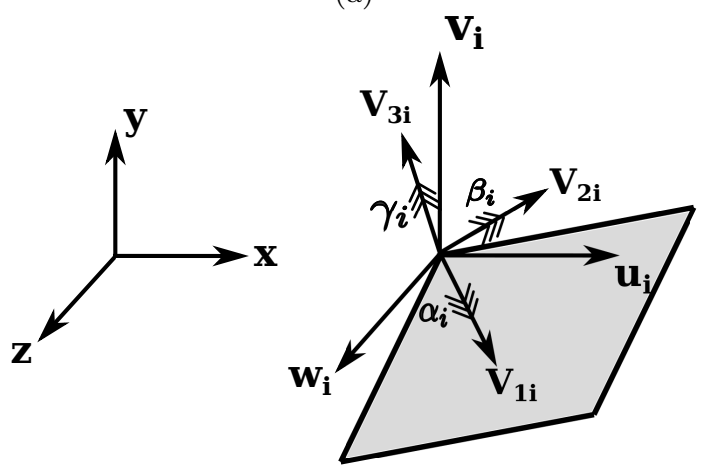

(b)

Fig. 1: A degenerated 4-noded Reissner-Mindlin shell element: (a) shell mid-surface (b) degrees of freedom and local coordinate system

The strain tensor $[\varepsilon]_{x y z}$ in the global cartesian system is defined according to Eq. (4) below.

$[\varepsilon]_{x y z}=\left[\begin{array}{llllll}\varepsilon_{x x} & \varepsilon_{y y} & \varepsilon_{z z} & \gamma_{x y} & \gamma_{y z} & \gamma_{z x}\end{array}\right]^{T}=\sum_{i=1}^{4}\left[\mathbf{B}_{i}^{\mathbf{u}}\right] d_{i}$

where $\left[\mathbf{B}_{i}^{\mathbf{u}}\right]$ is the $6 \times 6$ strain-displacement matrix at each shell node $i$. The detailed definition of matrix $\left[\mathbf{B}_{i}^{\mathbf{u}}\right]$ can be referred from Cook et al [23].

Remark 1 The drilling DOF $\gamma_{i}$ have no stiffness associated with them. Hence, when coplanar elements share a common structural node, the drilling rotation about the shell normal $V_{3 i}$ at that node is not resisted and the system matrix becomes singular. On the contrary when not all elements surrounding a structural node are coplanar, the normal rotation of any element at the shared node has a component which gets resisted by the bending stiffness of adjacent elements. This means that in flat-shell geometries, the drilling rotation DOFs $\gamma_{i}$ can be omitted from the list of overall structural DOFs. However when the shell geometry is curved, any such suppression of $\gamma_{i}$ would lead to an over-constrained model and unwarranted stiffening of the structure [23]. Keeping this in view, in this work all 6 DOFs $\left[u_{i}, v_{i}, w_{i}, \alpha_{i}, \beta_{i}, \gamma_{i}\right]$ are retained at nodes which (3)216 are shared by non-coplanar elements; they are however omitted for nodes shared by coplanar elements. 
To conveniently describe the kinematics of the shell 246 element, the following coordinate systems are intro-247 duced (Fig. 2), i.e.,

1. Global Cartesian coordinate-system $[x, y, z]$

2. Parametric coordinate-system $[\xi, \eta, \zeta]$ used for ${ }^{250}$ defining parametric space of the master element.

3. Shell-aligned local coordinate system $[1,2,3]$ based on mid-surface nodal vectors $\left[V_{1}, V_{2}, V_{3}\right]$ which are used to define the directions of rotational DOFs $\{\alpha, \beta, \gamma\}$.

4. Convective coordinate system $[r, s, t]$ in which MITC4+ modifications are performed. This can be given as $r=g_{1} /\left|g_{1}\right|, s=g_{2} /\left|g_{2}\right|, t=g_{3} /\left|g_{3}\right|$. Here, $g_{i}=\mathbf{x}_{, \zeta_{i}}$ are the tangent vectors to the shell-surface at any arbitrary point having position vector $x$, where $\zeta_{i} \in\{\xi, \eta, \zeta\}$ represents the parametric directions.

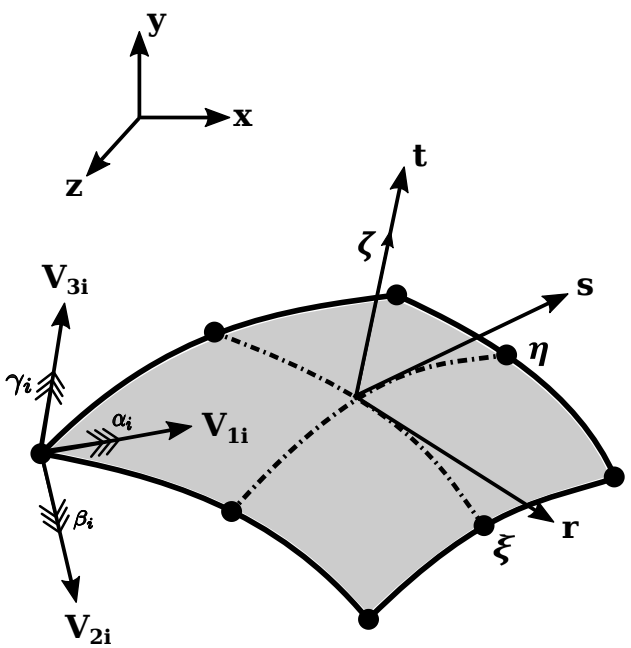

Fig. 2: Illustration of the different coordinate systems used in the formulation of the Reissner-Mindlin shell $_{260}$ element

\section{$2.3 \mathrm{MITC} 4 / \mathrm{MITC} 4+$ formulations}

In this section, the modified formulations for the transverse shear strain components based on the MITC4+ approach $[26,39]$ are briefly presented. The 4-noded flat shell element shown in Fig. 2 is considered, with it's convected and shell-aligned local coordinate systems represented by $[r, s, t]$ and $[1,2,3]$, respectively.

In the original MITC4 formulations [26], the transverse shear strains $\varepsilon_{s t}$ and $\varepsilon_{r t}$ are considered constant along the edges perpendicular to the $r$ and $s$ axes, respectively (Fig. 3a). Furthermore, instead of using the displacement based interpolations shown in Eq. (4), the transverse shear strain components at any arbitrary point inside the element are interpolated based on the strain values at a pre-defined set of tying points $\{A, B, C, D\}$ ( Fig. 3a) using Eq. (5).

$\varepsilon_{r t}=\frac{1}{2}(1+\eta) \varepsilon_{r t}^{(A)}+\frac{1}{2}(1-\eta) \varepsilon_{r t}^{(B)}$

$\varepsilon_{s t}=\frac{1}{2}(1+\xi) \varepsilon_{s t}^{(C)}+\frac{1}{2}(1-\xi) \varepsilon_{s t}^{(D)}$

The transverse shear strains at these tying points, i.e., $\left\{\varepsilon_{r t}^{(A)}, \varepsilon_{r t}^{(B)}, \varepsilon_{s t}^{(C)}, \varepsilon_{s t}^{(D)}\right\}$, are calculated using the standard approach in Eq. (4)

$\varepsilon_{r t}^{(T P)}=\left(\varepsilon_{r t}\right)_{\text {at TP using DI }}$

$\varepsilon_{s t}^{(T P)}=\left(\varepsilon_{s t}\right)_{\text {at TP using DI }}$

where $T P \in\{A, B, C, D\}$ denotes the tying points, and $D I$ denotes the direct displacement-based interpolation analogous to Eq. (4).

Similarly, in the MITC4+ formulations the membrane strain components $\left\{\varepsilon_{r r}, \varepsilon_{s s}, \varepsilon_{r s}\right\}$ are interpolated using Eq. (5) using the membrane tying points $\{A, B, C, D, E\}$ shown in Fig. 3b. The detailed expressions are omitted herein and can be found in [39].

\subsection{Coordinate transformations}

To formulate the local element matrices and the constitutive relations, the strain tensor in Eq. (4) must be transformed into the shell-aligned local coordinate system $[1,2,3]$ using the strain-transformation matrix $\mathcal{T}_{\varepsilon}$ according to Eq. (7)

$[\varepsilon]_{123}=\left[\begin{array}{llllll}\varepsilon_{11} & \varepsilon_{22} & \varepsilon_{33} & \gamma_{12} & \gamma_{23} & \gamma_{13}\end{array}\right]^{T}=\mathcal{T}_{\varepsilon}\left[\begin{array}{l}\varepsilon \\ ]_{x y z}\end{array}\right.$

A general definition for $\mathcal{T}_{\varepsilon}$ involving straintransformation between any two arbitrary coordinate systems is provided in Appendix B for completeness.

The assumed strains introduced in Eq. (5) are defined in the convected coordinate system $[r, s, t]$, whereas the strains in Eq. (7) are expressed with respect to the shell-aligned local system $[1,2,3]$. Hence, to impose the MITC4+ modification, the shell-aligned local strains $[\varepsilon]_{123}$ must be first transformed into the convective strains $[\varepsilon]_{r s t}$. Due to the planar geometry of the 4-noded Mindlin shell elements, the in-plane directions for both coordinate systems $[r, s]$ and $[1,2]$ are co-planar, but rotated with respect to each other. The rotation for transverse shear strains $\left[\gamma_{13}, \gamma_{23}\right]^{T}$ into the convected coordinates $[r, s, t]$ is performed according to Eq. (8)

$\left[\begin{array}{ll}\gamma_{r t} & \gamma_{s t}\end{array}\right]^{T}=[R]\left[\begin{array}{ll}\gamma_{13} & \gamma_{23}\end{array}\right]^{T}$ 


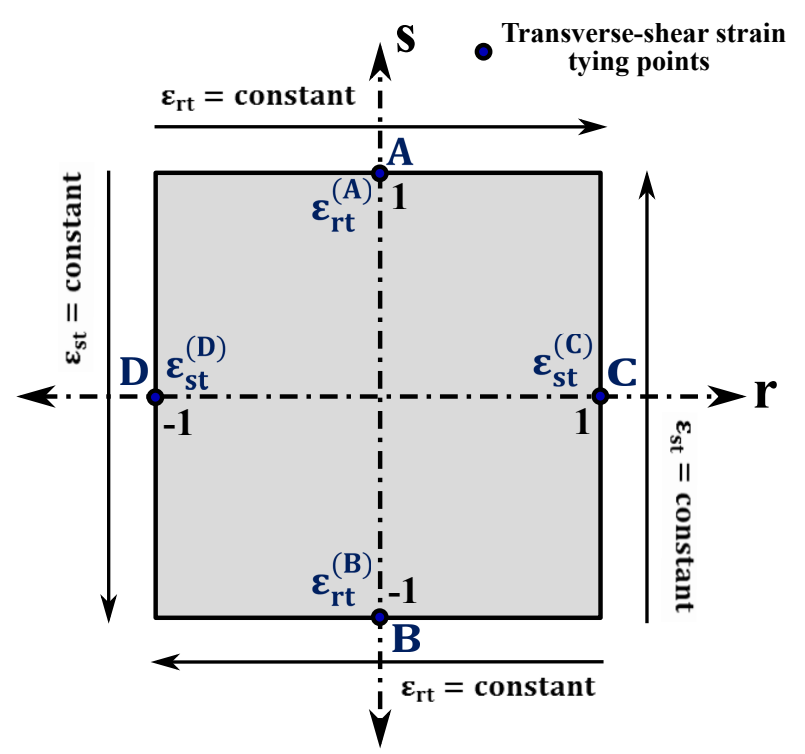

(a)

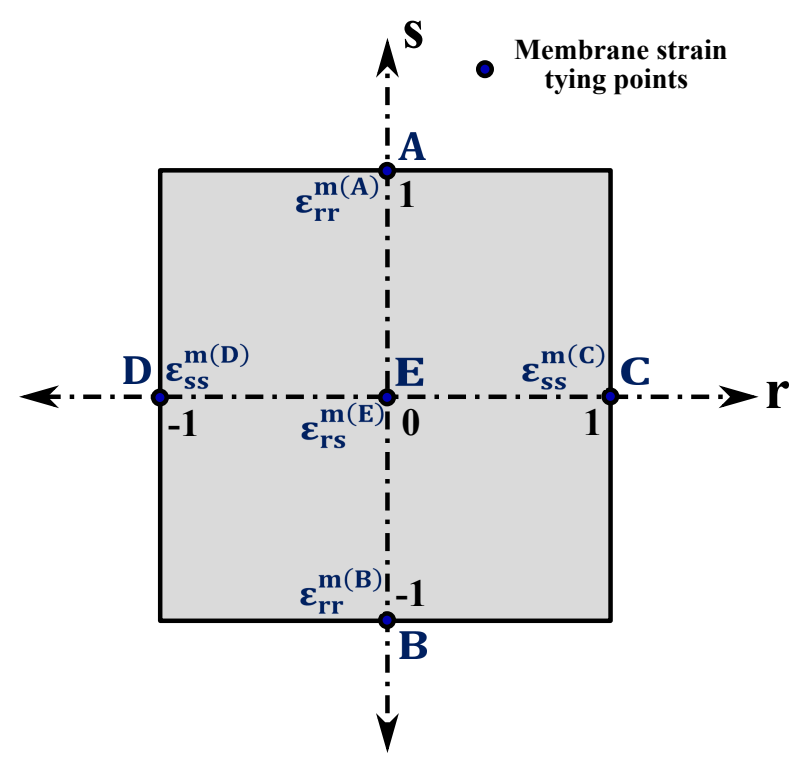

(b)

Fig. 3: Location of tying points used for assumption of (a) transverse-shear strains [26] (b) membrane strains within MITC4+ approach [39]

where

$[R]=\left[\begin{array}{cc}\sin \beta & -\sin \alpha \\ -\cos \beta & \cos \alpha\end{array}\right]^{-1}$.

In Eq. (9), $\alpha$ and $\beta$ are the angles between the $r$ and $V_{1}$ axes and $s$ and $V_{1}$ axes respectively.

The in-plane convective strain components277 $\left[\varepsilon_{r r}, \varepsilon_{s s}, \gamma_{r s}\right]$ is derived according to Eq. (10)

$\left[\varepsilon_{r r}, \varepsilon_{s s}, \gamma_{r s}\right]^{T}=\left[\mathcal{T}_{\varepsilon}^{\prime}\right][\varepsilon]_{123}$

where $[\varepsilon]_{123}$ is provided in Eq. (7). The transformation matrix $\mathcal{T}_{\varepsilon}^{\prime}$ is directly derived from $\mathcal{T}_{\varepsilon}$ in Appendix B using only the elements of the $1^{s t}, 2^{\text {nd }}$, and $4^{\text {th }}$ rows of $\mathcal{T}_{\varepsilon}$ that correspond to the in-plane strain components $\left[\varepsilon_{r r}, \varepsilon_{s s}, \gamma_{r s}\right]$.

After performing the MITC4+ modifications on the convective transverse shear strains $\left\{\gamma_{s t}, \gamma_{r t}\right\}$ and inplane membrane strains $\left\{\varepsilon_{r r}, \varepsilon_{s s}, \gamma_{r s}\right\}$, the total convected strain tensor $[\varepsilon]_{r s t}$ is transformed back into the shell-aligned local coordinate system $[\varepsilon]_{123}$ by applying the inverse of linear transformations shown in Eq. (8)-(10).

The overall shell-aligned local strain tensor can then be expressed according to Eq. (11).

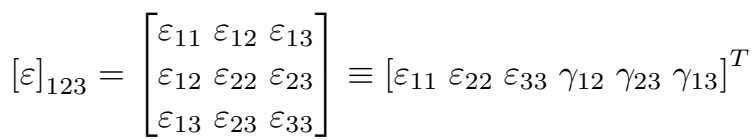

In the MITC4+ shell element, plane-stress assump-281 tions hold, i.e. the out-of-plane tensile stress $\sigma_{33}=0 \mathrm{in}_{282}$ the shell-aligned local coordinate system $[1,2,3]$. Hence, the expression for the out-of-plane tensile strain $\varepsilon_{33}$ is derived according to Eq. (12)

$\varepsilon_{33}=-\frac{\nu}{1-\nu}\left(\varepsilon_{11}+\varepsilon_{22}\right)$

where $\nu$ is the material Poisson's ratio. We further drop the subscript for local strains $[\varepsilon]_{123}$, and denote it as $[\varepsilon]$ for the remainder of this paper.

As discussed in Sec. 2, the translational DOFs $\left[u_{i}, v_{i}, w_{i}\right]$ are defined with respect to the the global Cartesian vectors $[x, y, z]$. However, the rotational DOFs $\left[\alpha_{i}, \beta_{i}, \gamma_{i}\right]$ are defined in the direction of shelllocal vectors $\left[V_{1}, V_{2}, V_{3}\right]$. Therefore, the local DOF vector $d_{l o c_{i}}=\left[u_{i}, v_{i}, w_{i}, \alpha_{i}, \beta_{i}, \gamma_{i}\right]$ is transformed to the global coordinate system according to Eq. (13) below

$d_{\text {glob }}=\left[\mathcal{T}_{\text {rot }}\right]^{T} d_{\text {loc }}$

with,

$\mathcal{T}_{\text {rot }}=\left[\begin{array}{cccccc}1 & 0 & 0 & 0 & 0 & 0 \\ 0 & 1 & 0 & 0 & 0 & 0 \\ 0 & 0 & 1 & 0 & 0 & 0 \\ 0 & 0 & 0 & l_{1} & m_{1} & n_{1} \\ 0 & 0 & 0 & l_{2} & m_{2} & n_{2} \\ 0 & 0 & 0 & l_{3} & m_{3} & n_{3}\end{array}\right]$

where $d_{g l o b}=\left[u_{x i}, v_{y i}, w_{z i}, \theta_{x i}, \theta_{y i}, \theta_{z i}\right]$ is the global vector of DOF and the expressions for the direction cosines $\left\{l_{i}, m_{i}, n_{i}\right\}$ with $i \in\{1,2,3\}$ are provided in B. 


\section{Constitutive phase-field model}

Griffith's theory of brittle fracture [32] derives from the assumption that the total potential energy of a fractured solid is additively decomposed into the bulk strain energy depending on the elastic deformations and the crack surface energy (Eq. (14))

$$
\Pi(\mathbf{u}, \Gamma)=\int_{\Omega} \psi_{e}(\varepsilon(\mathbf{u})) d \Omega+\int_{\Gamma_{c}} \mathcal{G}_{c} d \Gamma_{c}-\mathcal{W}_{\text {ext }}
$$

with, $\mathcal{W}_{\text {ext }}=\int_{\Omega} \mathbf{b} \cdot \mathbf{u} d \Omega+\int_{\partial \Omega} \mathbf{t} \cdot \mathbf{u} d \partial \Omega$

In Eq. (14), and also Fig. 4, $\mathbf{u}$ is the displacement vector ${ }^{286}$ at any arbitrary point within the domain $\Omega, \mathbf{b}$ and $\mathbf{t}^{287}$ represent the body forces within $\Omega$ and surface-traction ${ }^{28}$ forces on external boundary $\partial \Omega$ respectively, $\Gamma_{c}$ is the internal discontinuous boundary, $\psi_{e}$ is the elastic energy density and $\mathcal{G}_{c}$ is the critical fracture energy density. The linearised strain tensor $\varepsilon(\mathbf{u})$ is

$\varepsilon(\mathbf{u})=\frac{\nabla \mathbf{u}+\nabla^{T} \mathbf{u}}{2}$
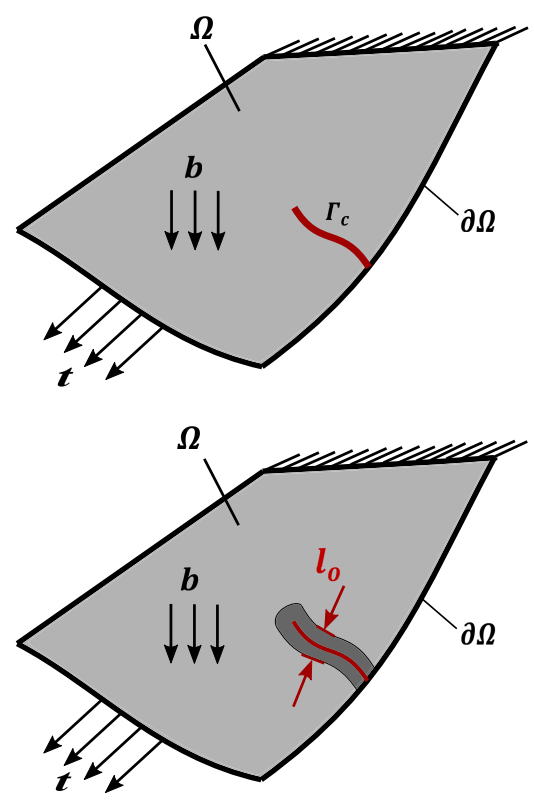

Fig. 4: Illustration of general shell-domain $\Omega$ containing (a) Internal sharp crack, and (b) Diffused crack, and subjected to body force $\mathbf{b}$ and surface traction forces $\mathbf{t}$

In the variational phase-field formulation, the sharp crack surface energy term in Eq. (14) is replaced by the regularized volume integral of a diffuse crack term shown in Eq. (16), i.e.,

$\int_{\Gamma_{c}} \mathcal{G}_{c} d \Gamma_{c} \approx \int_{\Omega} \mathcal{G}_{c} \gamma(\phi, \nabla \phi) d \Omega$

where, $\phi \in[0,1]$ is the phase-field variable. For a quadratic fracture surface energy approximation introduced in Ambrosio and Tortorelli $[6,5]$, the phase-field function $\gamma(\phi, \nabla \phi)$ assumes the following form, i.e.,

$\gamma(\phi, \nabla \phi)=\left[\frac{(\phi-1)^{2}}{4 l_{o}}+l_{o}|\nabla \phi|^{2}\right]$

where $l_{o}$ is the length-scale parameter controlling the width of phase-field diffusion zone. Using the functional definition of Eq. (17) it is straight-forward to show that $\phi=0$ and $\phi=1$ correspond to the fully-cracked and fully-intact states of the material, respectively.

As a crack evolves, the elastic strain energy and induced stresses of the solid must decrease to compensate for the fracture energy required to generate new crack surfaces. This degradation mechanism is achieved by means of a degradation function $g(\phi) \in[0,1]$ so that the elastic strain energy becomes

$\psi_{e}(\varepsilon, \phi)=g(\phi) \psi_{e}(\varepsilon)$.

Combining Eqs. (14)-(18), the following expression for the regularized potential energy of a cracked solid is obtained

$$
\begin{array}{r}
\Pi(\varepsilon, \phi, \nabla \phi)=\int_{\Omega}\left[g(\phi) \psi_{e}(\varepsilon)+G_{c} \gamma(\phi, \nabla \phi)\right] d \Omega \\
-\int_{\Omega} b_{i} u_{i} d \Omega-\int_{\partial \Omega} t_{i} u_{i} d \partial \Omega
\end{array}
$$

with $u_{i}, b_{i}$ and $t_{i}$ as the vector components of displacement $\mathbf{u}$, body-force $\mathbf{b}$ and surface traction force t respectively. Eq. (19) corresponds to the phase-field model with an isotropic energy split; this however results also in cracks evolving under pure compression.

To address the issue of non-physical crack evolution under pure compression, phase-field models based on an anisotropic energy-splitting have been proposed, see, e.g., $[8,41,3]$. In the current work, we employ the spectral decomposition of the strain tensor as introduced in Miehe et al [41] to facilitate comparisons with published results. To effectively impose plane-stress assumptions and calculate the in-plane and out-of-plane contributions of the strain energy density accurately, an additional 2-D strain tensor $[\varepsilon]^{\prime}$ comprising only in-plane strain components $\left[\varepsilon_{11}, \varepsilon_{22}, \varepsilon_{12}\right]$ is defined, i.e.,

$[\varepsilon]^{\prime}=\left[\begin{array}{ccc}\varepsilon_{11} & \varepsilon_{12} & 0 \\ \varepsilon_{12} & \varepsilon_{22} & 0 \\ 0 & 0 & \varepsilon_{33}\end{array}\right]$ with, $\varepsilon_{33}=\frac{-\nu}{1-\nu}\left(\varepsilon_{11}+\varepsilon_{22}\right)$ 
The effective Cauchy stress vector is defined accord-313 ingly as

$\sigma=\left[\sigma_{11}, \sigma_{22}, \sigma_{33}, \tau_{12}, \tau_{23}, \tau_{13}\right]^{T}$

Remark 2 To effectively impose the plane-stress assumption after damage has initiated, the in-plane membrane stress components $\left[\sigma_{11}, \sigma_{22}, \tau_{12}\right]^{T}$ and their corresponding contributions to the total strain energy density must be calculated based on the 2-D strain tensor $\varepsilon^{\prime}$ in Eq. (20), whereas the out-of-plane components $\left[\tau_{23}, \tau_{13}\right]^{T}$ and their strain-energy contributions calculated using the complete 3 -D strain tensor $\varepsilon$ in Eq. (11). ${ }^{315}$ In addition, the out-of-plane tensile stress $\sigma_{33}$ can be ex- ${ }^{316}$ plicitly set to zero to achieve optimal convergence char- ${ }^{317}$ acteristics and ensure that the plane-stress assumptions ${ }^{318}$ hold even post-initiation of damage.

The tensile and compressive components of the 2-D and 3-D strain tensors $\left\{\varepsilon^{\prime}, \varepsilon\right\}$ defined in Eq. (20) and (11) respectively, can be obtained using Eqs. (22) and (23) below.

$\varepsilon=\sum_{i=1}^{3} \lambda_{i} \mathbf{n}_{i} \otimes \mathbf{n}_{i} \quad ; \quad \varepsilon^{\prime}=\sum_{i=1}^{3} \lambda_{i}^{\prime} \mathbf{n}_{i}^{\prime} \otimes \mathbf{n}_{i}^{\prime}$

$[\varepsilon]_{ \pm}=\sum_{i=1}^{3}\left\langle\lambda_{i}\right\rangle_{ \pm} \mathbf{n}_{i} \otimes \mathbf{n}_{i} ; \quad\left[\varepsilon^{\prime}\right]_{ \pm}=\sum_{i=1}^{3}\left\langle\lambda_{i}^{\prime}\right\rangle_{ \pm} \mathbf{n}_{i}^{\prime} \otimes \mathbf{n}_{i}^{\prime}$

where $\lambda_{i} / \lambda_{i}^{\prime}$ are eigenvalues (principal stretches), $\mathbf{n}_{i} / \mathbf{n}_{i}^{\prime}$ are eigenvectors (principal stretch directions), and $\left\{\varepsilon_{ \pm}, \varepsilon_{ \pm}^{\prime}\right\}$ are the tensile/compressive strain components for the strain tensors $\left\{\varepsilon, \varepsilon^{\prime}\right\}$ respectively. The expres ${ }^{319}$ sion $\langle\cdot\rangle_{ \pm}$denote Macaulay brackets $\langle\cdot\rangle_{ \pm}=[(\cdot) \pm|(\cdot)|] / 2{ }^{320}$ where $\left\langle\lambda_{i}\right\rangle_{ \pm}$and $\left\langle\lambda_{i}^{\prime}\right\rangle_{ \pm}$contain only positive/negative ${ }^{321}$ eigenvalues of the strain tensors $\left\{\varepsilon, \varepsilon^{\prime}\right\}$ respectively.

Based on the spectral strain decomposition for the 2-D strain tensor $\left[\varepsilon^{\prime}\right]$ in Eq. (23), we define the in-plane components of strain energy density $\psi^{I P}$ and its corresponding tensile/ compressive parts $\psi_{ \pm}^{I P}$ in Eq. (24)

$\psi_{e}^{I P}\left(\varepsilon^{\prime}, \phi\right)=g(\phi) \psi_{+}^{I P}\left(\varepsilon_{+}^{\prime}\right)+\psi_{-}^{I P}\left(\varepsilon_{-}^{\prime}\right)$

$\psi_{ \pm}^{I P}\left(\varepsilon_{ \pm}^{\prime}\right)=\frac{\lambda}{2}\left\langle\operatorname{tr}\left(\varepsilon^{\prime}\right)\right\rangle_{ \pm}^{2}+\mu \operatorname{tr}\left[\left(\varepsilon_{ \pm}^{\prime}\right)^{2}\right]$

with $\lambda$ and $\mu$ as the Lamé constants, and $\mathcal{I}$ as $2 \times 2$ identity tensor. The corresponding split stress tensor definitions $\sigma_{ \pm}^{I P}$ are provided in Eq. (25) as

$\sigma^{I P}=\left[\begin{array}{ll}\sigma_{11} & \tau_{12} \\ \tau_{12} & \sigma_{22}\end{array}\right]=g(\phi) \sigma_{+}^{I P}\left(\varepsilon_{+}^{\prime}\right)+\sigma_{-}^{I P}\left(\varepsilon_{-}^{\prime}\right)$

$\sigma_{ \pm}^{I P}\left(\varepsilon_{ \pm}^{\prime}\right)=\left[\begin{array}{ll}\left(\sigma_{11}\right)_{ \pm} & \left(\tau_{12}\right)_{ \pm} \\ \left(\tau_{12}\right)_{ \pm} & \left(\sigma_{22}\right)_{ \pm}\end{array}\right]=\lambda\left\langle\operatorname{tr}\left(\varepsilon^{\prime}\right)\right\rangle_{ \pm} \mathcal{I}+2 \mu\left(\varepsilon_{ \pm}^{\prime}\right)$
The stress tensor $\sigma^{I P}$ is expressed in Voigt notation as $\sigma^{I P}=\left[\begin{array}{lll}\sigma_{11} & \sigma_{22} & \tau_{12}\end{array}\right]^{T}$.

According to Eqs. (24) and (25), only the positive tensile parts of the strain energy density and the Cauchy stress tensor, resepctively are multiplied by the degradation function $g(\phi)$. In this work, we employ the quadratic degradation function originally introduced in Pham and Marigo [45], Miehe et al [42], i.e.,

$g(\phi)=\left(1-\eta_{r}\right) \phi^{2}+\eta_{r}$

where the parameter $\eta_{r}$ was first defined in Ambrosio and Tortorelli [5] and denotes the residual stiffness to prevent ill-conditioning of system matrices when damage has fully propagated.

To similarly obtain the out-of-plane Cauchy stress $\sigma^{O P}$ and corresponding strain energy density terms $\left\{\psi_{e}^{O P}, \psi_{ \pm}^{O P}\right\}$, the tensile/ compressive components of full 3-D strain tensor $[\varepsilon]$ provided in Eq. (23) are used, as shown in Eq. (27)

$\psi_{e}^{O P}(\varepsilon, \phi)=g(\phi) \psi_{+}^{O P}\left(\varepsilon_{+}\right)+\psi_{-}^{O P}\left(\varepsilon_{-}\right)$

$\psi_{ \pm}^{O P}\left(\varepsilon_{ \pm}\right)=2 \mu\left[\left(\varepsilon_{23}\right)_{ \pm}^{2}+\left(\varepsilon_{13}\right)_{ \pm}^{2}\right]$

and Eq. (28)

$\sigma^{O P}=\left[\begin{array}{c}\tau_{23} \\ \tau_{13}\end{array}\right]=g(\phi) \sigma_{+}^{O P}\left(\varepsilon_{+}\right)+\sigma_{-}^{O P}\left(\varepsilon_{-}\right)$

$\sigma_{ \pm}^{O P}\left(\varepsilon_{ \pm}\right)=\left[\begin{array}{l}\left(\tau_{23}\right)_{ \pm} \\ \left(\tau_{13}\right)_{ \pm}\end{array}\right]=2 \mu\left[\begin{array}{l}\left(\varepsilon_{23}\right)_{ \pm} \\ \left(\varepsilon_{13}\right)_{ \pm}\end{array}\right]$

, respectively, where $\left\{\left(\varepsilon_{23}\right)_{ \pm},\left(\varepsilon_{13}\right)_{ \pm}\right\}$are the transverse shear components in the tensile/ compressive 3-D strain tensors $\left\{\varepsilon_{ \pm}\right\}$previously defined in Eq. (23).

In the standard Mindlin shell theory, the transverse shear stresses along the shell thickness are not constant; rather they follow a parabolic distribution. To account for this effect, the transverse shear strains in Eq. (28) are scaled by a factor of $5 / 6$ as also highlihgted in Cook et al [23].

$\tau_{23}=(5 / 6) \tau_{23}$

$\tau_{13}=(5 / 6) \tau_{13}$

Based on the in-plane and out-of-plane contributions given in Eq. (24) and (27), the overall tensile and compressive components of the total strain energy density can be given as in Eq. (30).

$\psi_{ \pm}=\psi_{ \pm}^{I P}\left(\varepsilon_{ \pm}^{\prime}\right)+\psi_{ \pm}^{O P}\left(\varepsilon_{ \pm}\right)$

and hence, the expression for the total potential energy in Eq. (19) can be modified to naturally suppress crack 
growth in the regions under pure compression.

$$
\begin{aligned}
& \Pi(\varepsilon, \phi, \nabla \phi)=\int_{\Omega}\left[g(\phi) \psi_{+}\left(\varepsilon^{+}\right)+\psi_{-}\left(\varepsilon^{-}\right)+\right. \\
& \left.G_{c} \gamma(\phi, \nabla \phi)\right] d \Omega-\int_{\Omega} b_{i} u_{i} d \Omega-\int_{\partial \Omega} t_{i} u_{i} d \partial \Omega
\end{aligned}
$$

The strong form of the governing linear momentum and phase-field evolution equations are henceforth obtained by minimizing the total potential energy in $\mathrm{Eq}_{332}$ (31) with respect to the field variables $\{\mathbf{u}, \phi\}$.

$\nabla \boldsymbol{\sigma}+\mathbf{b}=0$, on $\Omega$

$\left(\frac{4 l_{0}(1-k) \psi_{+}}{\mathcal{G}_{c}}+1\right) \phi-4 l_{0}^{2} \Delta \phi=1$, on $\Omega$

where the boundary conditions satisfy,

$\mathbf{u}=\overline{\mathbf{u}}$, on $\partial \Omega_{u}$

$\frac{\partial \phi}{\partial x_{i}} n_{i}=0$, on $\partial \Omega_{\phi}$

\subsection{Effective material tangent operator}

The undamaged material elastic constitutive law for homogeneous materials is expressed in the local shell- aligned coordinate system $[1,2,3]$ as

$$
\begin{gathered}
\sigma=\mathbf{C}_{\mathbf{o}} \varepsilon \\
\Rightarrow\left[\begin{array}{c}
\sigma_{11} \\
\sigma_{22} \\
\sigma_{33} \\
\tau_{12} \\
\tau_{23} \\
\tau_{13}
\end{array}\right]=\left[\begin{array}{cccccc}
E^{\prime} & \nu E^{\prime} & 0 & 0 & 0 & 0 \\
\nu E^{\prime} & E^{\prime} & 0 & 0 & 0 & 0 \\
0 & 0 & 0 & 0 & 0 & 0 \\
0 & 0 & 0 & G & 0 & 0 \\
0 & 0 & 0 & 0 & 5 G / 6 & 0 \\
0 & 0 & 0 & 0 & 0 & 5 G / 6
\end{array}\right]\left[\begin{array}{c}
\varepsilon_{11} \\
\varepsilon_{22} \\
\varepsilon_{33} \\
\gamma_{12} \\
\gamma_{23} \\
\gamma_{13}
\end{array}\right]
\end{gathered}
$$

where $E^{\prime}=E /\left(1-\nu^{2}\right)$ with $E$ and $\nu$ as Young's modulus and Poison's ratio respectively, and $G=0.5 G /(1+$ $\nu)$ is the shear modulus of the material [23].

Eq. (36) is derived on the basis of a plane-stress state and indicates that the in-plane components of the elastic Cauchy stress $\left[\sigma_{11}, \sigma_{22}, \tau_{12}\right]^{T}$ are obtained only using the corresponding in-plane components of undamaged material tangent $\mathbf{C}_{\mathbf{o}}$, whereas the transverse shear stress components $\left[\tau_{23}, \tau_{13}\right]^{T}$ are obtained using out-of-plane shear components of $\mathbf{C}_{\mathbf{o}}$.

To achieve optimal convergence rates even with the modified stress definitions in Eq. (25) and (28), planestress assumptions must hold even when the material is undergoing damage. To achieve this, we consider a split of the damaged tangent stiffness matrix $\mathcal{C}_{d}$ into its corresponding components as shown in Eq. (37) and (40), which are based on in-plane $\left\{\sigma^{I P}, \varepsilon^{\prime}\right\}$ or out-ofplane $\left\{\sigma^{O P}, \varepsilon\right\}$ stresses and strains, respectively.

$\left[\mathcal{C}_{d}\right]^{I P}=\frac{\partial \sigma^{I P}}{\partial \varepsilon^{\prime}}=g(\phi)\left[\mathcal{C}_{d}\right]_{+}^{I P}+\left[\mathcal{C}_{d}\right]_{-}^{I P}$

where

$\left[\mathcal{C}_{d}\right]_{ \pm}^{I P}=\frac{\partial \sigma_{ \pm}^{I P}}{\partial \varepsilon^{\prime}}$

The in-plane material tangent operator $\left[\mathcal{C}_{d}\right]^{I P}$ can also be represented as the $4 \times 4$ tensor shown in Eq. (39).

$\left[\mathcal{C}_{d}\right]^{I P}=\left[\begin{array}{cccc}\mathcal{C}_{d}^{1111} & \mathcal{C}_{d}^{1122} & 0 & \mathcal{C}_{d}^{1112} \\ \mathcal{C}_{d}^{2211} & \mathcal{C}_{d}^{2222} & 0 & \mathcal{C}_{d}^{2212} \\ 0 & 0 & 0 & 0 \\ \mathcal{C}_{d}^{1211} & \mathcal{C}_{d}^{1222} & 0 & \mathcal{C}_{d}^{1212}\end{array}\right] ; \mathcal{C}_{d}^{i j k l}=\frac{\partial \sigma_{i j}^{I P}}{\partial \varepsilon_{k l}^{\prime}}$

The out-of-plane component of material tangent operator can be similarly given as Eqs. (40) and (41).

$\left[\mathcal{C}_{d}\right]^{O P}=\left[\begin{array}{cc}\mathcal{C}_{d}^{2323} & 0 \\ 0 & \mathcal{C}_{d}^{1313}\end{array}\right]=g(\phi)\left[\mathcal{C}_{d}\right]_{+}^{O P}+\left[\mathcal{C}_{d}\right]_{-}^{O P}$ 
$\left[\mathcal{C}_{d}\right]_{ \pm}^{O P}=\left[\begin{array}{cc}\frac{\partial\left(\tau_{23}\right)_{ \pm}}{\partial\left(\varepsilon_{23}\right)_{ \pm}} & 0 \\ 0 & \frac{\partial\left(\tau_{13}\right)_{ \pm}}{\partial\left(\varepsilon_{13}\right)_{ \pm}}\end{array}\right]$

where $\left\{\left(\tau_{23}\right)_{ \pm},\left(\tau_{13}\right)_{ \pm}\right\}$and $\left\{\left(\varepsilon_{23}\right)_{ \pm},\left(\varepsilon_{13}\right)_{ \pm}\right\}$are the tensile/ compressive components of the $3-\mathrm{D}$ transverse shear stresses and strains defined in Eq. (28) and (23) respectively.

The combined damaged material tangent tensor $\left[\mathcal{C}_{d}\right]$ can finally be expressed as

$\left[\mathcal{C}_{d}\right]_{6 * 6}=\left[\begin{array}{cc}{\left[\mathcal{C}_{d}\right]^{I P}} & \mathbf{0} \\ \mathbf{0}^{T} & {\left[\mathcal{C}_{d}\right]^{O P}}\end{array}\right]$

where $\mathbf{0}$ corresponds to the $2 \times 4$ null tensor.

\subsection{Crack driving force variation along shell-thickness}

The 3-D kinematics of Mindlin shell elements are de- ${ }^{370}$ fined with respect to the kinematics of the mid-surface. ${ }^{371}$ Furthermore, damage evolution as manifested by the $\mathrm{e}^{372}$ evolution of the phase field is obtained only at the mid- ${ }^{373}$ surface nodes as a 2-D field. Hence, achieving an accu- ${ }^{374}$ rate and realistic stress degradation along the thickness ${ }^{375}$ becomes a challenging task [see, e.g., 38].

Driven by the observation that, especially in thin ${ }_{377}$ shell structures, crack propagation through all thickness layers is often sudden and brutal, we employ a maxi- ${ }_{379}$ mum through the thickness driving force rule to control ${ }_{380}$ the evolution of the phase field. Within this setting, the ${ }_{381}$ crack driving forces are evaluated at each through the ${ }_{382}$ thickness integration point according to Eq. (34) as ${ }_{383}$

$\mathcal{D}_{i j}=\max _{\left(t>t_{0}\right)} \psi_{+, i j}$

where $i=1 \ldots n_{\text {thick }}$ and $j=1 \ldots n_{G P}$ with $n_{\text {thick }}$ denoting the number of thickness layers and $n_{G P}$ the number of integration points per layer, respectively. Hence, the crack-driving force is evaluated based on the $3-\mathrm{D}$ stress state at its individual integration point.

The crack-driving force at all thickness integration points corresponding to a particular mid-surface location is then set equal to the maximum of driving forces prevalent at those integration points and phasefield evolution Eq. (35) is integrated at each Gausspoint over the entire shell-element volume, thus causing phase-field (or damage) to evolve based on the max crack-driving force description. The procedure is schematically illustrated in Fig. 5 for the case of

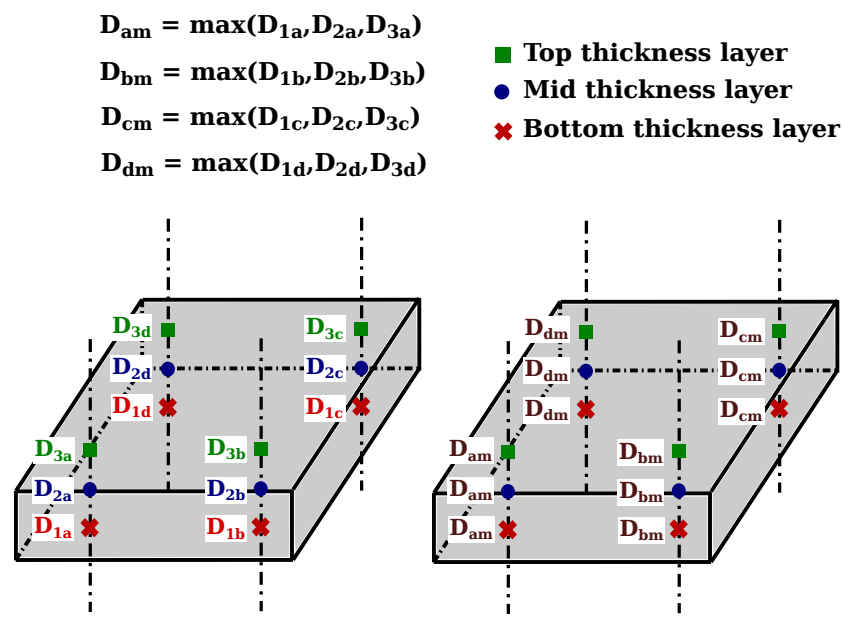

Fig. 5: Schematic illustration of the procedure employed to evaluate the crack-driving force $\mathcal{D}$ based on the maximum through the thickness rule employed. The case of 3 thickness layers and 4 integration point per thickness layer is considered.

$n_{\text {thick }}=3$ thickness layers and $n_{G P}=4$ integration points per layer.

Our extensive numerical experiments have shown that this assumption captures the physical cracking phenomena through the shell thickness and leads to highly accurate critical fracture strength predictions, especially during bending dominated failure scenarios, as also shown in the benchmark numerical examples.

Remark 3 To accurately capture the phase field variation through the thickness, in the case of multi-layered composite sections, see for e.g. [49], where a significant variation of the fracture toughness is expected, one would stack a number of shell elements along the thickness (see, e.g., [36, 59, 56]). Such aspects are beyond the scope of this work.

3.3 Discretization and solution procedure

The coupled strong-form evolution Eqs. (32) are discretized via a Galerkin approximation. The test $\mathcal{S}$ and weighting $\mathcal{W}$ function spaces for the displacement field are defined as

$\mathcal{S}_{\mathbf{u}}=\left\{\mathbf{u} \in\left(\mathcal{H}^{\mathbf{1}}(\boldsymbol{\Omega})\right)^{\mathbf{d}} \mid \mathbf{u}=\overline{\mathbf{u}}\right.$ on $\left.\partial \boldsymbol{\Omega}\right\}$

$\mathcal{W}_{\mathbf{u}}=\left\{\delta \mathbf{u} \in\left(\mathcal{H}^{\mathbf{1}}(\boldsymbol{\Omega})\right)^{\mathbf{d}} \mid \delta \mathbf{u}=\overline{\delta \mathbf{u}}\right.$ on $\left.\partial \boldsymbol{\Omega}\right\}$.

The corresponding spaces for the phase field are

$\mathcal{S}_{\phi}=\left\{\phi \in\left(\mathcal{H}^{1}(\Omega)\right)\right\}$

$\mathcal{W}_{\phi}=\left\{\delta \phi \in\left(\mathcal{H}^{1}(\Omega)\right)\right\}$. 
Multiplying the strong form Eqs. (32), integrating ${ }_{41}$ by parts and performing the necessary algebraic manipulations eventually leads to the the following convenient nodal residual form for the equilibrium equation at node $i$,

$$
\begin{aligned}
\mathcal{R}_{i}^{\mathbf{u}} & =\mathcal{F}_{\text {ext }}^{\mathbf{u}}-\mathcal{F}_{\text {int }}^{\mathbf{u}} \\
& =\int_{V} N_{i} b_{i} d V-\int_{V}\left[\mathcal{T}_{\text {rot }}\right]^{T}\left[\mathcal{T}_{\varepsilon} \mathbf{B}_{i}^{\mathbf{u}}\right]^{T} \sigma d V
\end{aligned}
$$

and the phase-field evolution equation at node $i$

$$
\begin{aligned}
\mathcal{R}_{i}^{\phi} & =-\mathcal{F}_{i n t}^{\phi}=\int_{V}\left(\frac{4 l_{0}(1-k) \mathcal{D}}{\mathcal{G}_{c}}+1\right) N_{i} \phi d V \\
& +\int_{V} 4 l_{0}^{2}\left[\mathbf{B}_{i}^{\phi}\right]^{T}\left[\mathbf{B}_{i}^{\phi}\right] \phi_{i} d V-\int_{V} N_{i} d V
\end{aligned},
$$

respectively. In Eqs. (48) and (49), $V$ is the element volume, $N_{i}$ is the 2-D shape function and $\left[\mathbf{B}_{i}^{\mathbf{u}}\right]$ is the strain-displacement matrix as expressed in Eq. (4), and $\left[\mathcal{T}_{\text {rot }}\right],\left[\mathcal{T}_{\varepsilon}\right]$ are the rotation and strain-transformation tensors defined in Eqs. (13) and (7), respectively, which facilitate the calculation of the internal forces $\mathcal{F}_{\text {int }}^{\mathbf{u}}$ in the local shell coordinate system $[1,2,3]$ and their subsequent rotation into global $[x, y, z]$ system.

The explicit expressions for $N_{i}$ and $\left[\mathbf{B}_{i}^{\mathbf{u}}\right]$ can be obtained from [23]. Furthermore, $\left[\mathbf{B}_{i}^{\phi}\right]$ is defined with respect to shell-local system $[1,2,3]$ as shown in Eq. (50).

$\left[\mathbf{B}_{i}^{\phi}\right]=\left[N_{i, 1}, N_{i, 2}, N_{i, 3}\right]^{T}$

Remark 4 In practice, the components of $\left[\mathbf{B}_{i}^{\phi}\right]$ can be $_{413}^{412}$ effectively obtained by choosing the relevant compo-414 nents of locally transformed strain-displacement tensor ${ }_{415}$ $\left[\mathcal{T}_{\varepsilon} \mathbf{B}_{i}^{\mathbf{u}}\right]$. Since in Mindlin shell theory, the kinematics of $\mathrm{f}_{416}$ the shell-element is represented using 2-D shape func- ${ }_{417}$ tions at the mid-surface, $N_{i, 3}$ can be effectively set as as $_{18}$ zero.

Assembling the contributions from each element shown in Eqs. (48) and (49) into the overall residual vectors $\mathcal{R}^{\mathbf{u}}$ and $\mathcal{R}^{\phi}$, the solution $\{\mathbf{u}, \phi\}$ to the com- $^{-421}$ bined system of equations (32) can be obtained by setting $\mathcal{R}^{\mathbf{u}} \rightarrow \mathbf{0}$ and $\mathcal{R}^{\phi} \rightarrow \mathbf{0}$.

In the current work, the solution is obtained us-423 ing the staggered or alternating minimization approach h $24^{4}$ based on [41]. To ensure accuracy of the obtained solu-425 tion, either both equations must be solved using stag-426 gered iterations [2] or the analysis must be solved using ${ }_{427}$ small incremental steps [41].

\subsection{Integration procedure}

For the MITC4+ shell element analyzed in the current work, a full-integration technique is employed with 4 Gauss integration points defined at each parametric thickness layer within the element. The integral expressions in Eqs. (48) and (49) are expressed in terms of parametric coordinates $[\xi, \eta, \zeta]$ according to Eq. (51)

$\int_{V}(\mathcal{I}) d V=\int_{-1}^{1} \int_{-1}^{1} \int_{-1}^{1}(\mathcal{I}) \operatorname{det}[\mathrm{J}] d \xi d \eta d \zeta$

where $\mathcal{I}$ is evaluated at each integration point through the shell-volume and the definition for Jacobian $[J]$ is provided in Appendix A. The in-plane integration over $\{\xi, \eta\}$ within each thickness layer $\zeta$ is performed using the Gauss-integration rule,

$\int_{-1}^{1} \int_{-1}^{1}(\mathcal{I}) \operatorname{det}[J] d \xi d \eta=\sum_{i=1}^{4}\left(\mathcal{I}_{i}\right) \operatorname{det}[J]_{i} w_{i}$

where $i \in\{1,2,3,4\}$ are the in-plane integration points and $w_{i} \in\{1,1,1,1\}$ are the weights associated with each of these points. The out-of-plane integration for all thickness layers is performed using the Simpson's rule, which can be expressed as in Eq. (53) for any integrand $\mathcal{I}^{\prime}$.

$$
\int_{-1}^{1} \mathcal{I}^{\prime} d \zeta=\frac{\Delta h}{3}\left(\mathcal{I}_{0}^{\prime}+2 \mathcal{I}_{1}^{\prime}+4 \mathcal{I}_{2}^{\prime}+2 \mathcal{I}_{3}^{\prime}+\ldots+\ldots+\mathcal{I}_{n}^{\prime}\right)
$$

where $\Delta h=2 / n$, and $\left\{\mathcal{I}_{0}^{\prime}, \mathcal{I}_{1}^{\prime}, \ldots, \mathcal{I}_{n}^{\prime}\right\}$ are the values of the integrand $\mathcal{I}^{\prime}$ evaluated at the different shellthickness layers $\zeta \in[-1,1]$ starting with the value of $\mathcal{I}_{0}^{\prime}$ at the bottom-most layer $\zeta=-1$.

While performing through-thickness integration of the phase-field evolution equation (49), the value of crack-driving force $\mathcal{D}$ at any Gauss-point within a specific thickness layer is obtained based on the maximum crack-driving force rule detailed in Sec. 3.2 and Fig. 5.

\section{Numerical examples}

In all the test cases examined in this Section, a displacement controlled analysis has been employed. Unless explicitly stated, a one-pass staggered (alternating minimization) approach with a very small time-increment size $\left(1 . e^{-06}-1 . e^{-05}\right)$ has been used for the solution of the coupled displacement- phase-field problem, and the residual stiffness $\eta_{r}$ is set to 0 . 
4.1 Notched square plate subjected to in-plane tension ${ }_{457}$

The standard benchmark of the notched square plate $e_{459}$ shown in Fig. 6 under tension is examined herein ${ }_{460}$ The material properties considered are $E=210 \mathrm{GPa}_{461}$ $\nu=0.3$, and $\mathcal{G}_{c}=0.0027 \mathrm{kN} / \mathrm{mm}$. The mesh-size $\mathrm{is}_{462}$ $h_{e}=0.0025 \mathrm{~mm}$ in the central strip where the crack is s63 $_{463}$ expected to propagate and the length scale parameter is ${ }_{464}$ $l_{o}=0.0075 \mathrm{~mm}$. A displacement control analysis is performed with an equilibrium tolerance of $t_{0 l} l_{u}=1 . e^{-08}{ }^{465}$

It is interesting to note that the length-scale param- ${ }^{467}$ eter $l_{0}$ adopted by Miehe et al [41] is twice the size of ${ }^{68}$ $l_{0}$ used by Borden et al [16]. This implies that the for ${ }^{469}$ mulation detailed in [41] requires the minimum value ${ }^{470}$ of $l_{0}$ to be at-least twice the mesh-size $h_{e}\left(l_{0} \geq 2 h_{e}\right)$, whereas on the other hand, the minimum value of $l_{0}$ should be $l_{0} \geq h_{e}$ for the formulations provided in [16]. Indeed both the definitions of $l_{0}$ are equivalent, and one must be careful while appropriately choosing the value ${ }_{473}$ of $l_{0}$ when comparing results from the two formulations ${ }_{474}$ The current work uses the formulations from [16], $\operatorname{and}_{475}$ hence the definition $l_{0} \geq h_{e}$ consistently hold for all the $e_{476}$ numerical simulations performed in this paper.

The resulting crack-path and load-displacement re-- ${ }^{478}$ sponse are shown in Fig. 7 and Fig. 8, respectively. ${ }^{49}$ The crack initiates at a critical fracture force $\mathcal{F}_{\text {crit }}={ }^{480}$ $0.7052 k N$. Both the crack-path and the fracture force ${ }^{481}$ prediction are in perfect agreement with the results $\mathrm{re}^{-482}$ ported in the literature [see, e.g., 42].

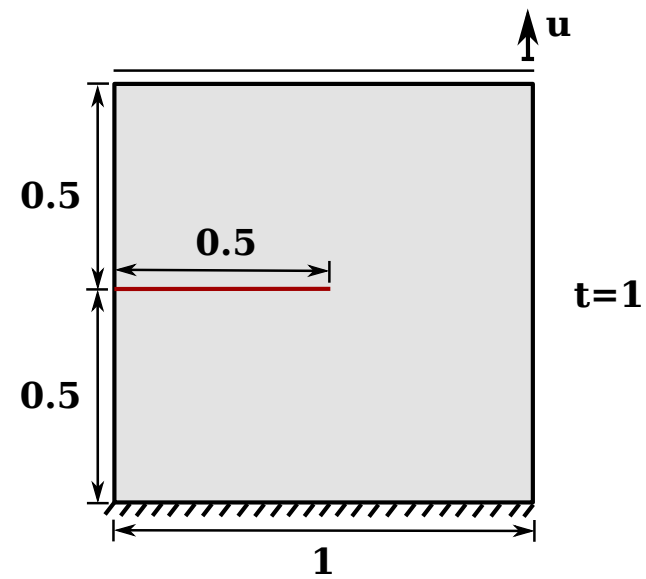

Fig. 6: Geometry and boundary conditions for square plate with horizontal notch subjected to in-plane tension (All dimensions in $\mathrm{mm}$ )
4.2 Notched square plate subjected to in-plane shear

The square plate specimen examined in Sec. 4.1 is subjected to horizontal in-plane tractions. Due to the nature of the loading and boundary conditions in this case, the specimen attains a bi-axial strain state which leads to the propagation of crack at an angle of $45^{\circ}$ to the horizontal direction. An equilibrium tolerance of $t_{0 l}=1 . e^{-06}$ is used for the displacement controlled analysis. Fig. 10 and Fig. 11 display the development of crack with each subsequent load-increment and the load-displacement response, respectively. The predicted crack-path and the critical fracture load $\mathcal{F}_{\text {crit }}=$ $0.5248 k N$ are in good agreement with the results reported in [41].

\subsection{1-D beam subjected to transverse bending}

A simply-supported rectangular plate subjected to a uniformly distributed pressure over the entire top face is considered as shown in Fig. 12. The aim of this example is to verify the proposed formulation predictions under bending-dominated fracture scenarios. The material and fracture properties are $E=1 . e 10 \mathrm{MPa}, \nu=0$, $\mathcal{G}_{c}=3 \mathrm{~N} / \mathrm{mm}$, and $l_{o}=0.01 \mathrm{~mm}$. The mesh is refined with $h_{e}=0.003 \mathrm{~mm}$ in the entire mid-span of the plate where the crack propagation is expected. The thickness of the beam $t=0.01 \mathrm{~mm}$ is very small in comparison to the other two plate-dimensions $(l=8 \mathrm{~mm}$ and $w=1$ $\mathrm{mm}$ ) so that the effects of transverse shear and membrane locking on the critical fracture characteristics can be monitored.

The vertical displacement is monitored at the centre-node of the plate, and the total applied distributed load is analysed with $\operatorname{tol}_{u}=1 \cdot e^{-06}$. The crack initiates at the plate's mid-span which is also the location of maximum transverse deformation $u_{z}$, as shown in Fig. 13. The load-displacement response is shown in Fig. 14 where a brittle fracture response under pure bending is indeed recovered.

Since the Poisson's ratio is null, the transverse bending stiffness and the critical fracture loads should be identical to those predicted by the classical Euler/ Bernoulli beam theory. According to the Euler/ Bernoulli beam theory, the analytical elastic stiffness/length of the beam is established in Eq. (54) as

$k=P / \delta=\frac{384 E I}{5 l^{4}}$

where $\delta$ is the maximum transverse deformation obtained at the centre-span, $E$ is the Young's modulus, $I=w t^{3} / 12$ is the area moment of inertia for the beam, 

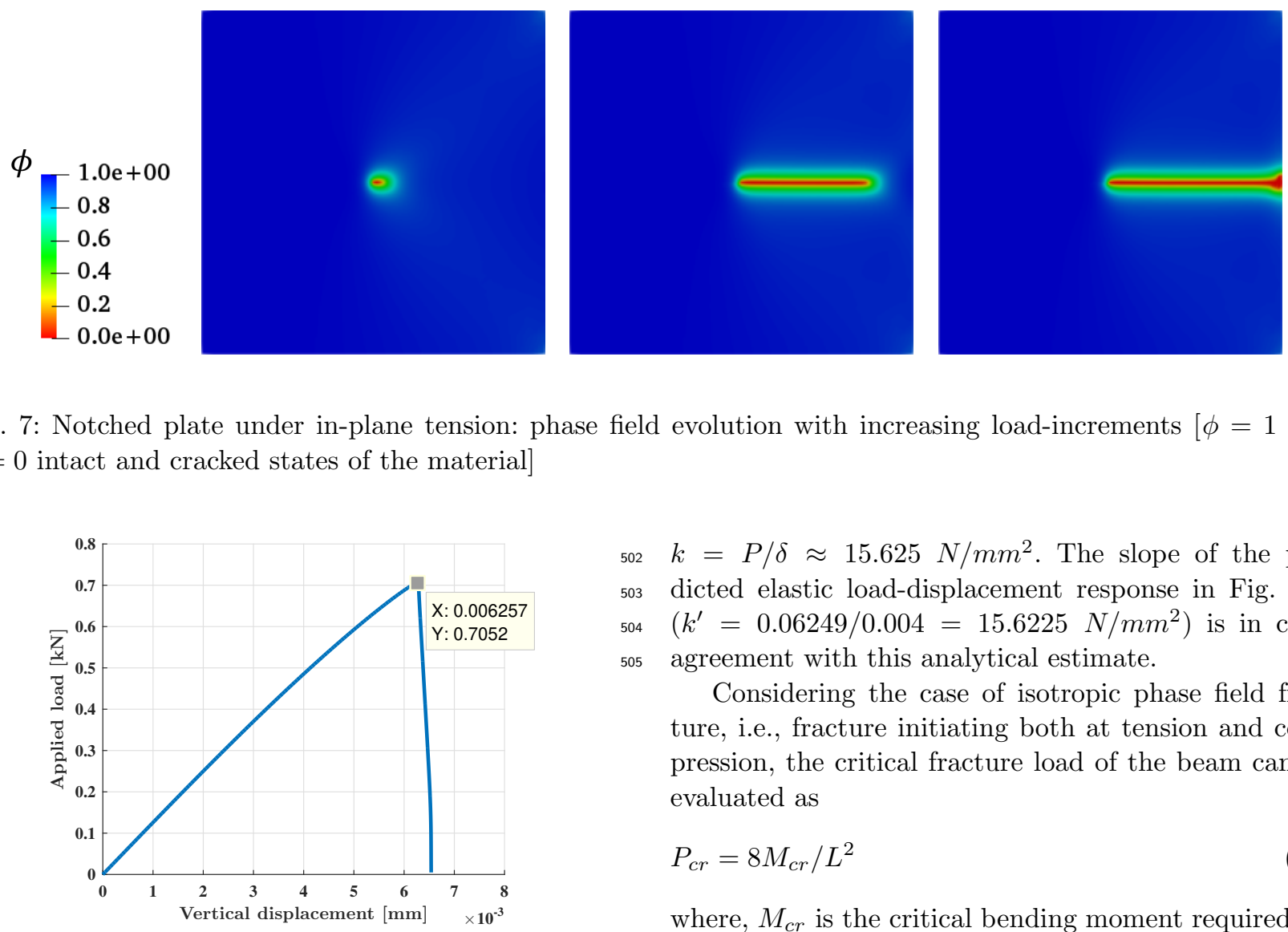

Fig. 7: Notched plate under in-plane tension: phase field evolution with increasing load-increments $[\phi=1$ and $\phi=0$ intact and cracked states of the material]

Fig. 8: Notched plate under in-plane tension: Loaddisplacement response

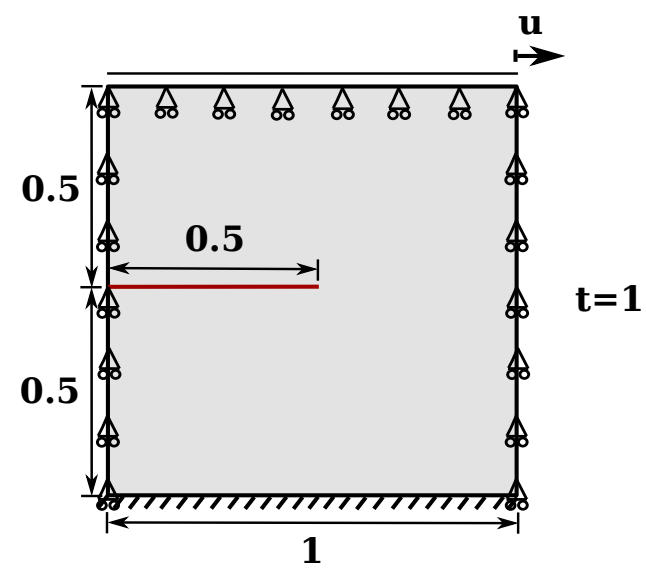

Fig. 9: Geometry and boundary conditions for square ${ }^{513}$ plate with horizontal notch subjected to in-plane shear ${ }^{514}$ (All dimensions in $\mathrm{mm}$ )

and $P=F / l$ is the total distributed applied load-518 /length on the beam with units in $N / m m$, wherein $F_{519}$ is the total applied load in $N$.

For the current case, the analytical elastic stiff-521 ness of the beam can be calculated using Eq. (54) as522
$k=P / \delta \approx 15.625 \mathrm{~N} / \mathrm{mm}^{2}$. The slope of the predicted elastic load-displacement response in Fig. 14b $\left(k^{\prime}=0.06249 / 0.004=15.6225 \mathrm{~N} / \mathrm{mm}^{2}\right)$ is in close agreement with this analytical estimate.

Considering the case of isotropic phase field fracture, i.e., fracture initiating both at tension and compression, the critical fracture load of the beam can be evaluated as

$P_{c r}=8 M_{c r} / L^{2}$

where, $M_{c r}$ is the critical bending moment required for crack initiation

$M_{c r}=\sigma_{c r} w t^{2} / 6$

and $\sigma_{c r}$ is the critical fracture stress. Based on derivations in [16], the critical fracture stress can be evaluated as Eq.(57).

$\sigma_{c r}=\frac{9}{16} \sqrt{\frac{E \mathcal{G}_{c}}{6 l_{o}}}$

For the given material and fracture properties, the critical stress in Eq. (57) is $\sigma_{c r}=3.9775 \cdot 10^{5} \mathrm{~N} / \mathrm{mm}^{2}$. This can be inserted into Eq. (55) to obtain the critical fracture load $P_{c r}=0.8286 \mathrm{~N} / \mathrm{mm}$.

Comparing the load-displacement responses in Fig. 14a, it is evident that the maximum crack-driving force description through thickness (detailed in Sec. 3.2) produces good agreement with the analytical fracture force estimated by Eq. (55) for the isotropic phase-field model. This reinstates the validity of the assumption that in thin shells, all transverse thickness layers at a given location would fracture simultaneously as soon as the crack is initiated in any one of these layers. Hence to incorporate this effect, the material stiffness degradation at that shell location must start as soon as the crack-driving force in any one of the associated thickness layers attains a critical limit. Such a description of 

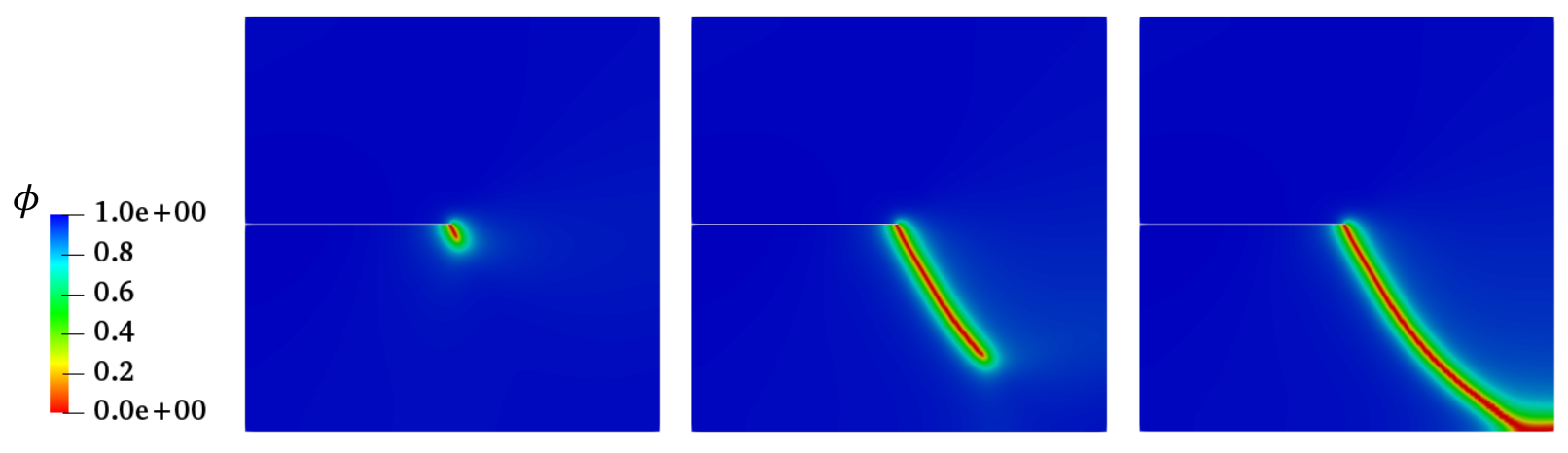

Fig. 10: Notched plate under in-plane shear: phase field evolution with increasing load-increments $[\phi=1$ and $\phi=0$ represent intact and cracked states of the material]

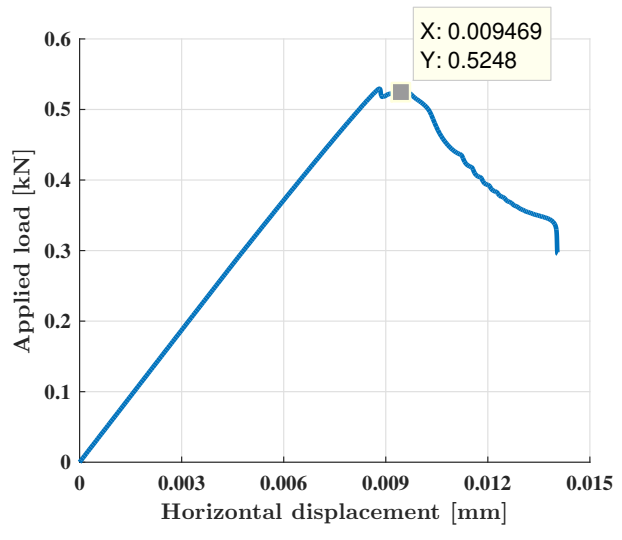

Fig. 11: Notched plate under in-plane shear: Loaddisplacement response

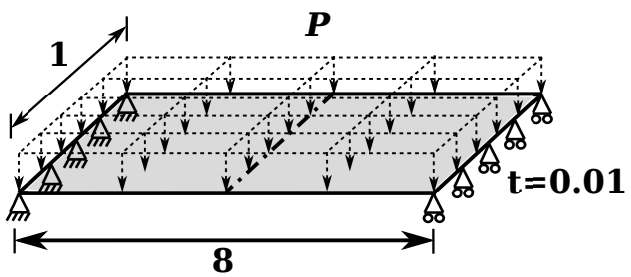

Fig. 12: Geometry and boundary conditions for rectan-535 gular 1-D beam subjected to transverse unidirectional bending under uniformly distributed pressure load $P_{536}$ (All dimensions in $\mathrm{mm}$ ) crack-driving force $\mathcal{D}$ enables a $3-\mathrm{D}$ description of $\operatorname{crack}_{539}$ topology and stress-degradation effects, albeit using $\mathrm{a}_{540}$ 2-D phase-field, refer to Sec. 3.2 for details.

Solving the phase-field evolution Eq. (32) using the 542 spectral split proposed in [41] and with the same crack-543 driving force definition (Fig. 5) results in the load-544 displacement response in Fig. 14b. The corresponding $5_{54}$ critical fracture load is higher than the one provided by546 the isotropic model as the in this case material degra-547 dation occurs only on the part of the shell undergoing tension. The accuracy of the predicted critical force for the spectral-split case [41] is verified against the analytical estimates and XFEM results in Sec. 4.4.
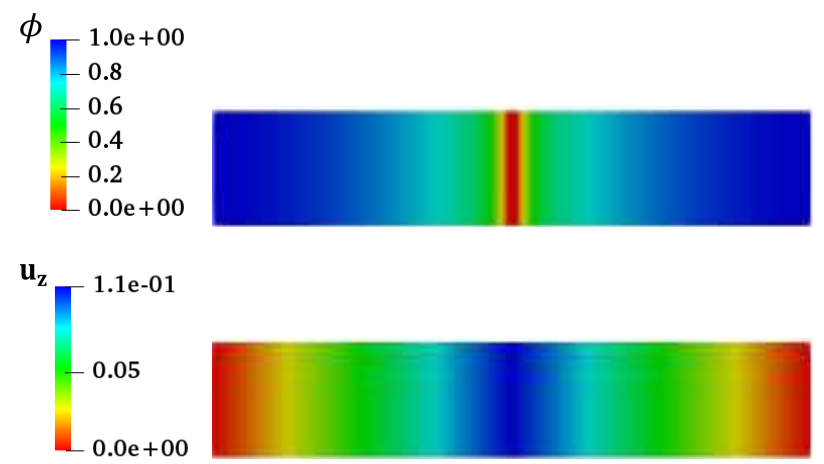

Fig. 13: 1-D beam under transverse unidirectional bending: Plan-view of (a) Crack-path $\phi$ and, (b) Vertical displacement $u_{z}$ represented at the shell mid-surface $[\phi=1$ and $\phi=0$ represent intact and cracked states of the material]

4.4 Regtangular plate with a through crack subjected to pure bending moments

The rectangular plate specimen with a through crack shown in Fig. 15 is subjected to pure bending moments on its opposite edges and the accuracy of predicted peak moments are compared with the corresponding analytical values obtained using the stress-intensity factors in [58]. This example has been examined previously in Rouzegar and Mirzaei [53], where a comparison between SIFs obtained with XFEM and the analytical SIFs was performed. Herein, In this example, we attempt a comparison between the critical fracture loads predicted by 


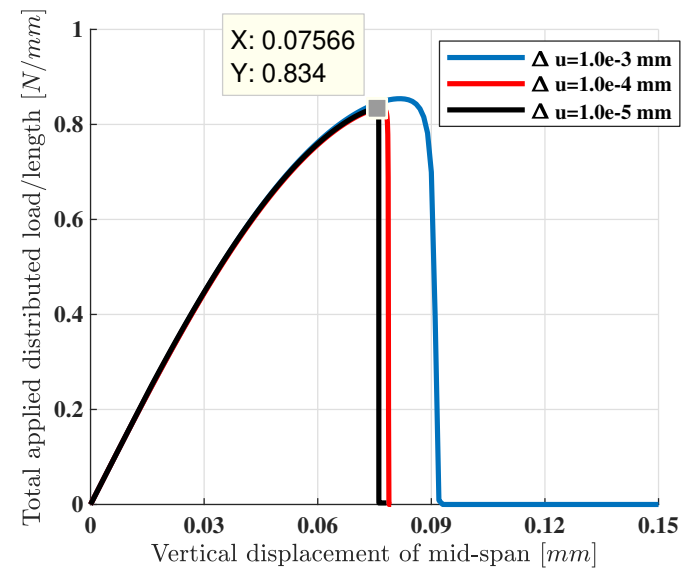

(a)

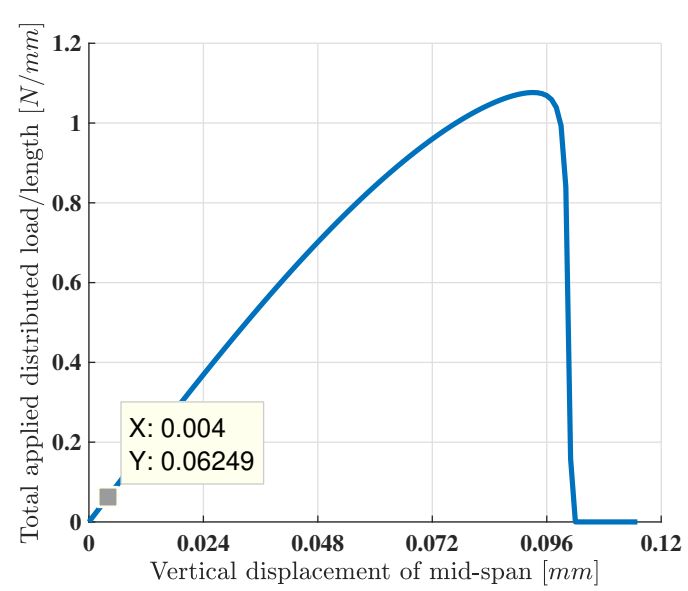

(b)

Fig. 14: 1-D beam under transverse unidirectional bending: Load-displacement response at beam's centre-node for a) Isotropic phase-field formulations b) Anisotropic phase-field formulations with spectral decomposition [41]

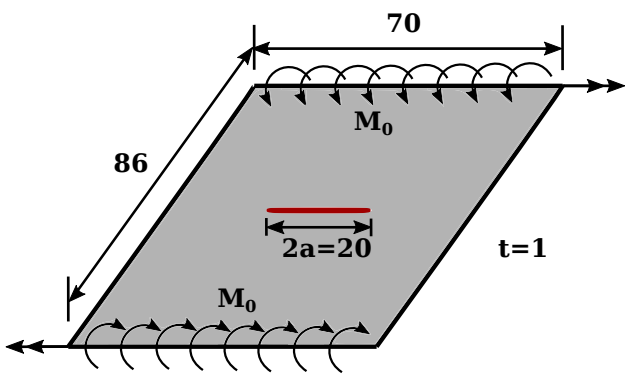

Fig. 15: Geometry and boundary conditions for the rectangular plate subjected to pure bending moments (All dimensions in $\mathrm{mm}$ )

the proposed phase-field model and the analytical formulations provided in Sih et al [58]. The material properties are $E=210,000 \mathrm{MPa}$ and $\nu=0.33$.
The rotational increment $\Delta \theta_{X}$ is monitored at the top-right corner node, and the plate is analysed with respect to varying sizes of $\Delta \theta_{X}$ until the peak critical bending-moment is converged. An equilibrium tolerance of $t o l_{u}=1 . e^{-06}$ is used in each case. According to [58], the analytical expression for the critical stressintensity factor (SIF) for a centrally-cracked plate with infinite width and subjected to remotely applied pure bending moment is evaluated as

$$
\begin{aligned}
K_{1 c} & =\frac{6 M_{0, c r i t}}{t^{2}} \sqrt{a} ; K_{2 c}=0 \\
\Rightarrow K_{c} & =\sqrt{K_{1 c}^{2}+K_{2 c}^{2}}
\end{aligned}
$$

where $K_{c}$ is the equivalent critical SIF, $t$ is the plates54 thickness, $M_{0, \text { crit }}$ is the critical bending moment and $a_{555}$ is half-length of the central crack. The analytical valuess6 of critical SIF for this example is provided in [53] as

$$
K_{1 c}=189.74 \mathrm{MPa} \mathrm{mm}^{-1 / 2} \text {. }
$$

Assuming plane-stress conditions, the corresponding critical energy release rate $\mathcal{G}_{c}$ is

$\mathcal{G}_{c}=\frac{K_{c}^{2}}{E}=0.171435 \mathrm{~N} / \mathrm{mm}$.

Substituting the value of $K_{1 c}$ from Eq. (59) into (58) and considering the edge length $l=70 \mathrm{~mm}$, the critical bending moment/edge-length is derived as

$\frac{M_{0, c r i t}}{l}=\frac{K_{1 c} t^{2}}{6 l \sqrt{a}}=10.0002 \mathrm{~N}-\mathrm{mm} / \mathrm{mm}$

In our phase-field simulations, the mesh is refined in the central region with the element size $h_{e}=0.25 \mathrm{~mm}$ where the crack is expected to propagate. The lengthscale parameter and residual stiffness are chosen as $l_{0}=0.25 \mathrm{~mm}$ and $\eta_{r}=1.0 e^{-3}$, respectively. In the original variational formulation proposed by Bourdin et al [20], it was shown that the fracture energy is overestimated depending on the size of finite element discretization. To compensate for this amplification, an effective critical energy release rate was proposed for the purpose of phase-field simulations, see also [46].

$\mathcal{G}_{c}^{e f f}=\frac{\mathcal{G}_{c}}{1+\left(h_{e} / 4 l_{0}\right)}$

Considering $\mathcal{G}_{c}=0.171435 \mathrm{~N} / \mathrm{mm}, h_{e}=0.25 \mathrm{~mm}$ and $l_{0}=0.25 \mathrm{~mm}$, the effective critical energy release rate $\mathcal{G}_{c}^{\text {eff }}$ for the current analysis is $\mathcal{G}_{c}^{\text {eff }}=$ $0.13715 \mathrm{~N} / \mathrm{mm}$.

The moment versus edge rotation response is illustrated in Fig. 16. The resulting crack topology is 
shown in Fig. 17. The crack originates simultaneously588 at both notch-tips and propagates horizontally toward\$589 the ends of the plate.

Furthermore, to demonstrate that the obtained re-591 sults are independent of the magnitude of chosen time-592 increments, a comparison of moment-rotation responses593 for varying sizes of moment-increments $\Delta M_{0}$ is per-594 formed in Fig. 16. The converged value for the critical moment/length in Fig. 16 is $M_{0, P F M}=10.83 \mathrm{~N}$ $\mathrm{mm} / \mathrm{mm}$, which is in close agreement with the analytical bending moment/length derived in Eq. (61). This ${ }_{596}$ example further establishes the validity of assumptions made in Sec. 3.2 for the phase-field model based ${ } n_{597}$ anisotropic spectral strain decomposition, and verifies ${ }_{598}$ the accuracy of the proposed phase-field formulations ${ }_{599}$ in characterising realistic bending-dominated fracture ${ }_{600}$ scenarios.

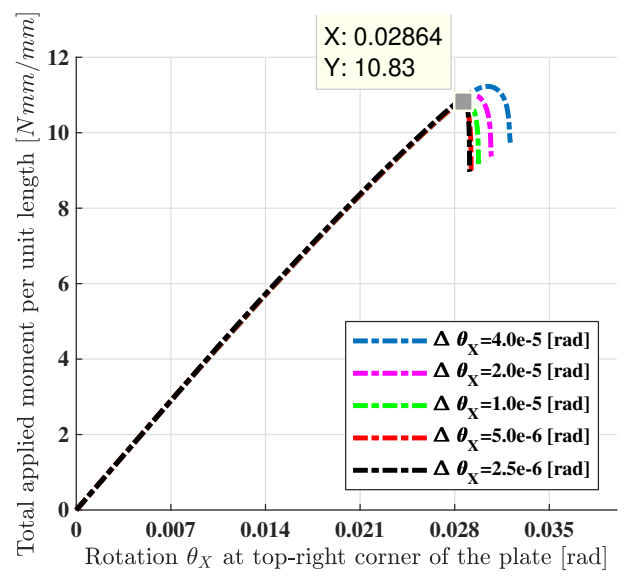

Fig. 16: Regtangular plate under pure-bending mo-617 ments: Moment vs edge-rotation response 4.5 Simply supported plate subjected to bi-directional ${ }^{623}$ bending loads

To demonstrate cracking phenomena under bi-526 directional bending loads, a simply supported plate627 with a uniformly distributed surface load is examined.628 The material and fracture properties are $E=1.9 e^{5_{629}}$ $\mathrm{MPa}, \nu=0.3, l_{o}=0.01 \mathrm{~mm}, G_{c}=0.295 \mathrm{~N} / \mathrm{mm}, 330$ and the boundary conditions are as shown in Fig. 18.631 The mesh is refined along the plate's diagonals witho32 $h_{e}=0.005 \mathrm{~mm}$. Only a quarter section of the plate is 633 analyzed due to symmetry. The quarter-section is sim-634 ply supported on the outer edges of the plate, whereas 35 the internal shared edges are subjected to symmetric636 boundary conditions. A uniformly distributed load is 637 applied over the entire top face until complete fracture of the plate, and the vertical displacement is monitored at the centre node of the plate. The analysis is run until a convergence tolerance of $t o l_{u}=1 . e^{-06}$ is reached.

The crack-path is shown in Fig. 19 which is consistent with the results reported previously in $[38,9]$. The load-displacement curve is illustrated in Fig. 20.

4.6 Cylinder with/without spherical closing cap subjected to uniform pressure loads

A cylindrical shell geometry with small axial notches placed on diametrically opposite ends and uniformly applied pressure load on its inner surface is considered. Owing to the problem symmetry across the xy and $x z$ planes, only the quarter part of the full cylinder is analyzed as shown in Fig. 21.

To examine the robustness of the approach, two different cases are examined, i.e. with and without a spherical cap at the two ends of the cylindrical shell. The latter is expected to give rise to crack branching at the spherical cap. The material and fracture properties are $E=7.0 e^{4} \mathrm{MPa}, \nu=0.3, l_{o}=0.125 \mathrm{~mm}$, $G_{c}=1.5 \mathrm{~N} / \mathrm{mm}$. The mesh is refined with the size $h_{e}=0.1 \mathrm{~mm}$ in all the cylindrical and spherical cap regions where the crack is expected to propagate. A displacement controlled analysis is performed with an equilibrium tolerance of $t_{o l}=1 \cdot e^{-05}$. For the cylinder specimen without spherical cap (Fig. 21a), the vertical circular arc BC is fixed along the $x$ and $z$ directions, whereas symmetric boundary conditions are imposed on horizontal edges $\mathrm{AB}, \mathrm{CD}$, and $\mathrm{AD}$. The specimen with spherical closing cap (Fig. 21b) is subjected to symmetric boundary conditions on all free edges, i.e. the vertical circular arc AD towards the notch is subjected to $\mathrm{y}$-symmetric and horizontal edges $\mathrm{AB}, \mathrm{BC}$ and $\mathrm{CD}$ are subjected to z-symmetric boundary conditions. The example demonstrates the capability of proposed phase-field formulations in simulating damage for thin curved geometries which displays significant membrane as well as transverse shear locking.

The responses between the total applied pressure load and the displacement-norm measured at the notchtip are compared in Fig. 22 for both the uncapped and capped specimens.

The crack-path at increasing load-increments for the uncapped and capped cylinders are shown in Figs. 23 and 24 , respectively. In the former case, the crack initiates at the notch-tip and propagates along the longitudinal direction of the shell. In the latter, the specimen demonstrates a similar response (Fig. 24), however, in this case the crack initiates at a slightly lower 

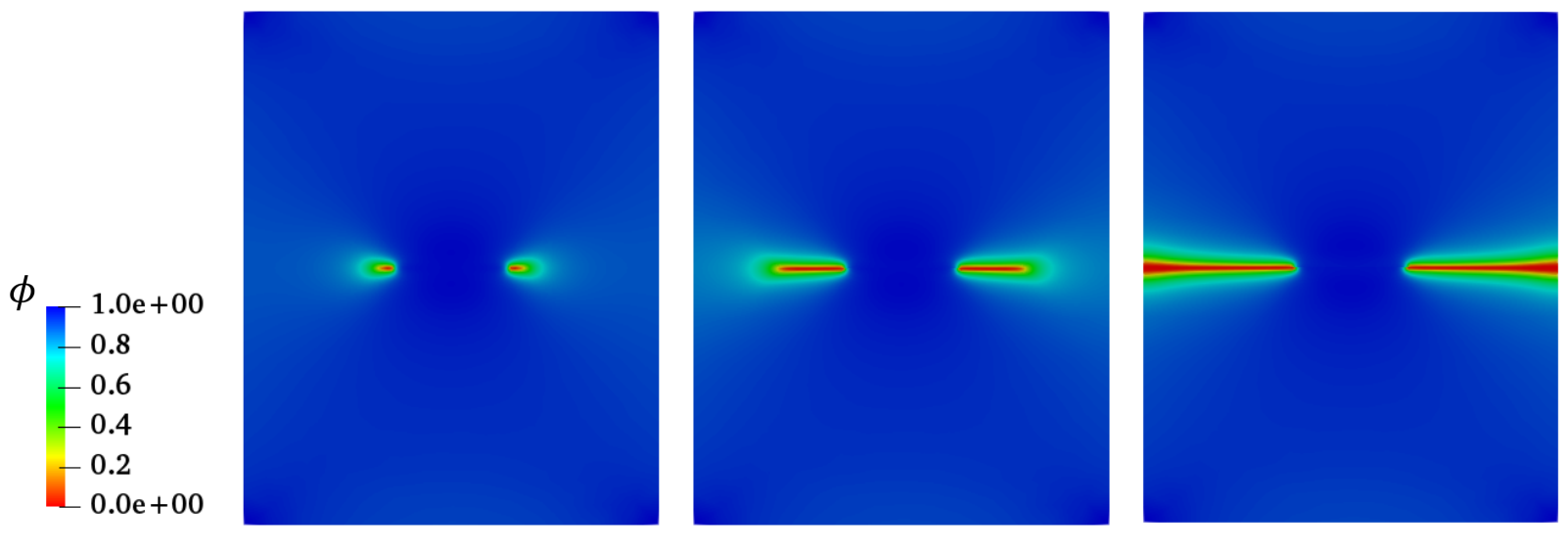

Fig. 17: Regtangular plate under pure-bending moments: phase field evolution with increasing load-increments $[\phi=1$ and $\phi=0$ represent intact and cracked states of the material]

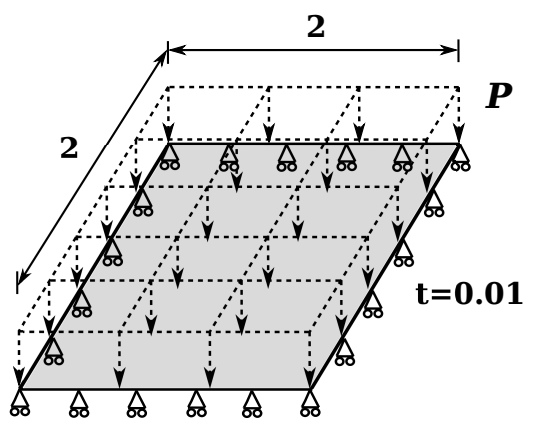

(a) Full-plate

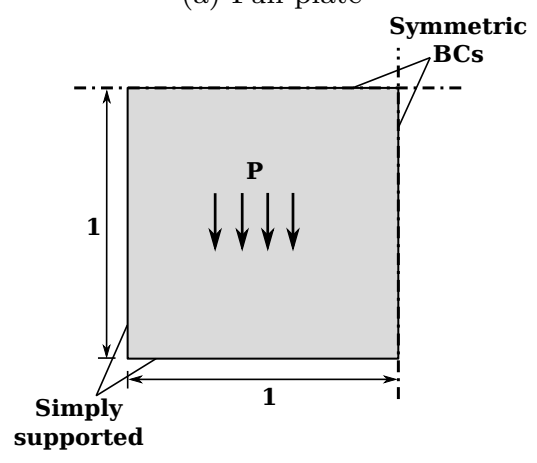

(b) Quarter-part of the plate
Fig. 18: Geometry and boundary conditions of simply- ${ }^{665}$ supported plate subjected to bidirectional bending un-666 der uniformly distributed pressure load $P$ (All dimen-667 sions in $\mathrm{mm}$ ) critical fracture load (Fig. 22). Over the spherical capo73 region, the crack first propagates linearly, but subse-674 quently splits into two symmetric crack branches; these675 further evolve simultaneously.
4.7 Assymetric hyperboloid subjected to uniform internal pressure

To further demonstrate the robustness of proposed formulations in analysing curved shell problems, an assymetric hyperboloid geometry is considered which is subjected to a uniform internal pressure applied in the direction normal to its surface. The thin-shell assumptions apply as the thickness of the geometry $t=0.1$ $\mathrm{mm}$ is significantly smaller than the other dimensions of the tower. A notch is introduced at the mid-height along the longitudinal direction of the shell. Due to the model symmetry only half part of the complete model as shown in Fig. 25 is analysed. To reduce the effect of bending at the boundary, the hyperboloid geometry is supported by an elastic shell structure, displayed as ABFE in Fig. 25 in which the evolution of phase-field (or damage) is restricted.

The material and fracture parameters for the hyperboloid are $E=210 \mathrm{GPa}, \nu=0.3, \mathcal{G}_{c}=0.0027$ $\mathrm{kN} / \mathrm{mm}, l_{o}=0.75 \mathrm{~mm}$, and a uniform mesh size with $h_{e}=0.5 \mathrm{~mm}$ is used. The material properties for the elastic base-support is $E=21000 \mathrm{GPa}, \nu=0.3$ with the Young's modulus chosen as 100 times higher than the hyperboloid.

Furthermore, the translational DOFs at the bottommost part of the elastic base-support is completely fixed $\left(u_{x}=u_{y}=u_{z}=0\right)$ while the rotational DOFs are kept free. For the curved side-edges $\mathrm{BC}$ and $\mathrm{AD}$, z-symmetric boundary conditions are imposed whereas the top-edge CD is unrestrained. The internal distributed load is applied only on the hyperboloid region EFCD in the direction of outward-pointing normals to its surface. The elastic support ABFE is unloaded. The radial displacement is monitored at the bottom notch-tip shown by $\mathrm{P}$ in Fig. 25, and $t_{0} l_{u}=1 . e^{-05}$. The crack initiates at the 

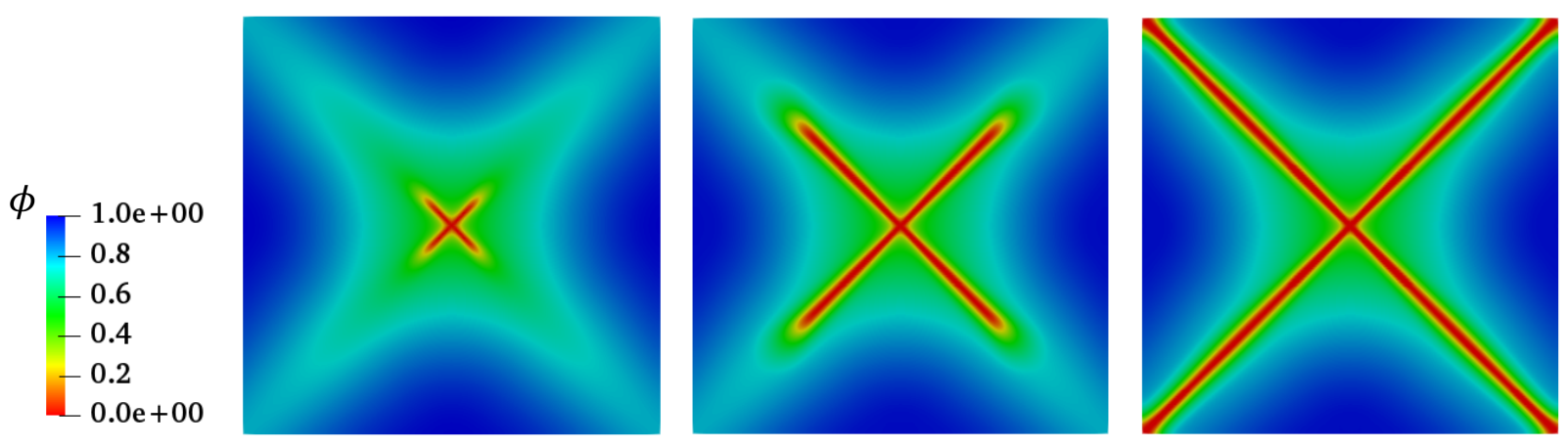

Fig. 19: Simply-supported plate under bidirectional bending: phase field evolution with increasing load-increments $[\phi=1$ and $\phi=0$ represent intact and cracked states of the material] (Full-plate assembled for better visualization)

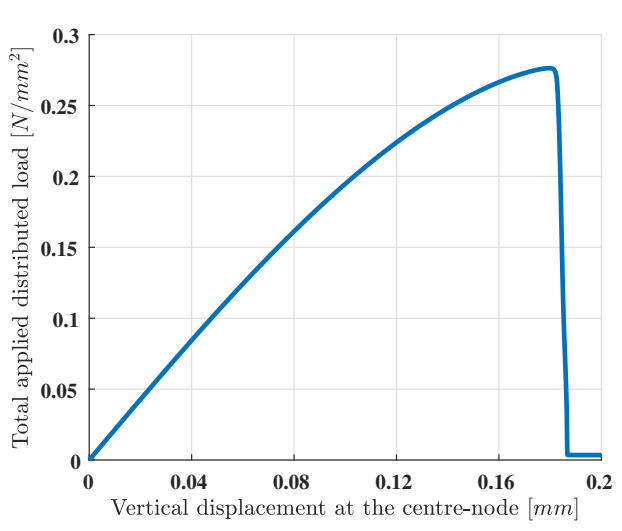

Fig. 20: Simply-supported plate under bidirectional bending: Load-displacement response at the centre node of the plate bottom notch-tip $\mathrm{P}$ as shown in Fig. 26, and propagates vertically downwards followed by a second branch that initiates at the top notch-tip Q. The two cracks propagate simultaneously and crack-branching is eventually observed at the bottom crack due to the shell-curvature at which point the shell loses all bearing capacity. The response between the vertical $z$-displacement at the bottom notch-tip $\mathrm{P}$ and the total applied load is shown in Fig. 27.

\section{Conclusion}

A phase-field driven shell element formulation is presented for of brittle fracture in Reissner-Mindlin shells. We employ an MITC4+ approach to alleviate shear and membrane locking. Our method is based on the assumption of a maximum through the thickness crack driving force rule definition. Considering an anisotrpic split for damage evolution, we impose the plane stress assump-695 tions directly on the tangent constitutive matrix; thiso96

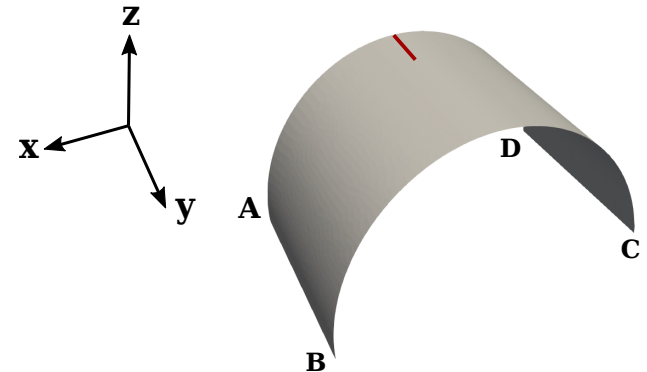

(a)

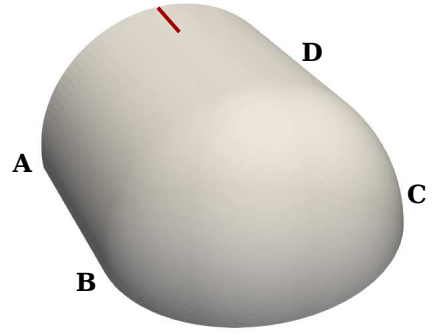

(b)
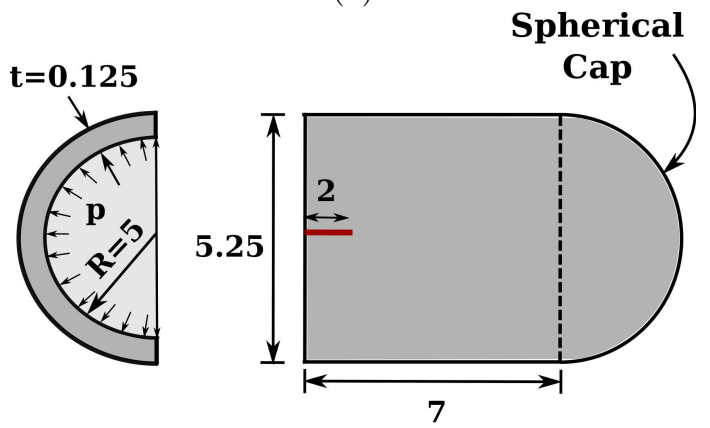

(c)

Fig. 21: Geometry and boundary conditions of cylindrical shell with notch (a) without (b) with spherical cap at the end, and subjected to uniform internal pressure $p$ (All dimensions in $\mathrm{mm}$ )

approach has been found to provide optimum convergence rates. 


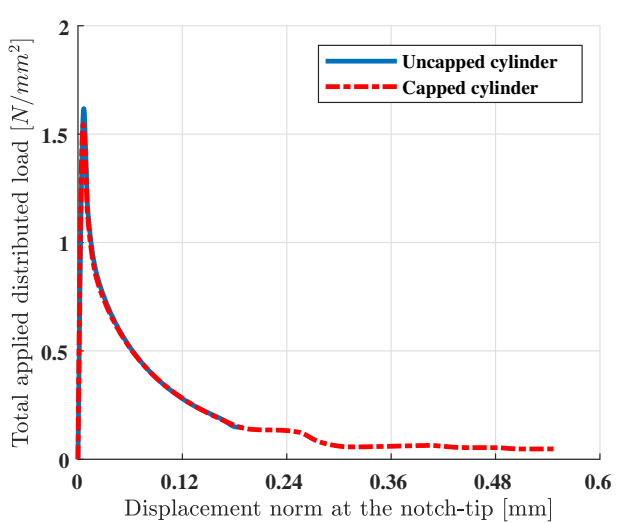

Fig. 22: Notched cylinder with/without spherical cap ${ }^{33}$ under uniform internal pressure: Applied pressure load ${ }^{39}$ vs norm of the displacement $\mathbf{u}_{n o r m}=\sqrt{u_{x}^{2}+u_{y}^{2}+u_{z}^{2740}}$ measured at the notch-tip

The accuracy of the proposed model is demon-744 strated by a set of illustrative numerical examples. Our ${ }^{75}$ solutions are verified against the analytical estimates ${ }^{76}$ both in the isotropic and anisotropic phase field case..$^{747}$ The validity of the proposed model is further estab- ${ }^{748}$ lished by obtaining realistic and accurate fracture pre $^{-749}$ dictions in curved shell geometries, which display sig $^{-750}$ nificant membrane and transverse shear locking due to $0^{751}$ the coupling of membrane and bending deformations. ${ }^{752}$

The inclusion of rotational degrees of freedom in the ${ }^{753}$ MITC4+ formulation would naturally raise an imple- ${ }^{-754}$ mentational challenge vis-à-vis the modelling of multi-755 layered composite profiles where delamination is a pos-756 sible failure mode $[22,48]$. In this case, coupling with ${ }^{757}$ e.g., a cohesive zone model would require the evaluation ${ }^{758}$ of displacements at the interface based on the Reissner- ${ }^{-759}$ Mindlin kinematical assumptions and the definition of ${ }^{60}$ multi-point constraints coupling the degrees of freedom ${ }^{761}$ associated with the shell and cohesive elements at the ${ }^{762}$ interface [54, 55, 21].

763

Whereas the proposed model highlights the capa $^{764}$ bilities of brittle fracture phase field modelling to har ${ }^{765}$ ness the advantages of MITC4+ formulations, research ${ }^{766}$ should be directed to account for more complex re$^{-767}$ sponses as,e .g., the case of finite strain ductile fracture. ${ }^{768}$ In the near future, we aim to extend the capabilities of ${ }^{69}$ the proposed phase-field model in simulating diverse ${ }^{70}$ anisotropic fracture scenarios.

\section{Acknowledgement}

The authors would like to acknowledge the funding re--776 ceived from the European Union's Horizon 2020 re-777 search and innovation programme under the Mariezs
Skłodowska-Curie SAFE-FLY project, grant agreement No. 721455 .

\section{References}

1. Aldakheel F, Hudobivnik B, Hussein A, Wriggers $P$ (2018) Phase-field modeling of brittle fracture using an efficient virtual element scheme. Computer Methods in Applied Mechanics and Engineering 341:443-466

2. Ambati M, Gerasimov T, De Lorenzis L (2015) Phase-field modeling of ductile fracture. Computational Mechanics 55(5):1017-1040

3. Ambati M, Gerasimov T, De Lorenzis L (2015) A review on phase-field models of brittle fracture and a new fast hybrid formulation. Computational Mechanics 55(2):383-405

4. Ambati M, Kruse R, De Lorenzis L (2016) A phasefield model for ductile fracture at finite strains and its experimental verification. Computational Mechanics 57(1):149-167

5. Ambrosio L, Tortorelli V (1992) On the approximation of free discontinuity problems. BULLETIN OF THE ITALIAN MATHEMATICAL UNION B

6. Ambrosio L, Tortorelli VM (1990) Approximation of functional depending on jumps by elliptic functional via t-convergence. Communications on Pure and Applied Mathematics 43(8):999-1036

7. Amiri F, Millán D, Shen Y, Rabczuk T, Arroyo M (2014) Phase-field modeling of fracture in linear thin shells. Theoretical and Applied Fracture Mechanics 69:102-109

8. Amor H, Marigo JJ, Maurini C (2009) Regularized formulation of the variational brittle fracture with unilateral contact: Numerical experiments. Journal of the Mechanics and Physics of Solids 57(8):12091229

9. Areias P, Rabczuk T (2013) Finite strain fracture of plates and shells with configurational forces and edge rotations. International Journal for Numerical Methods in Engineering 94(12):1099-1122

10. Barenblatt GI (1962) The mathematical theory of equilibrium cracks in brittle fracture. Advances in applied mechanics 7:55-129

11. Bathe KJ (2006) Finite element procedures. KlausJurgen Bathe

12. Bathe KJ, Dvorkin EN (1986) A formulation of general shell elements - the use of mixed interpolation of tensorial components. International Journal for Numerical Methods in Engineering 22(3):697-722

13. Belytschko T, Black T (1999) Elastic crack growth in finite elements with minimal remeshing. Interna- 

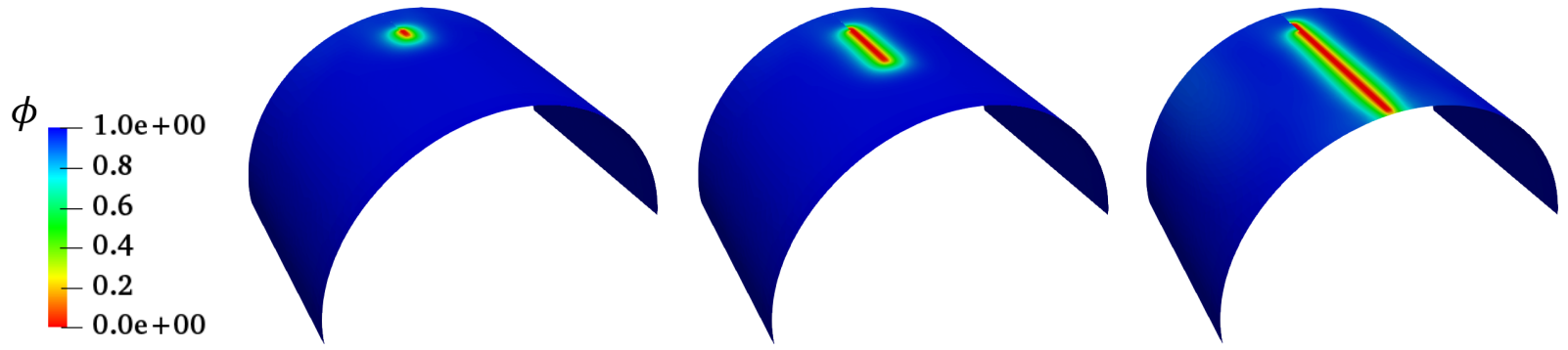

Fig. 23: Notched cylinder under uniform internal pressure: phase field evolution with increasing load-increments $[\phi=1$ and $\phi=0$ represent intact and cracked states of the material]
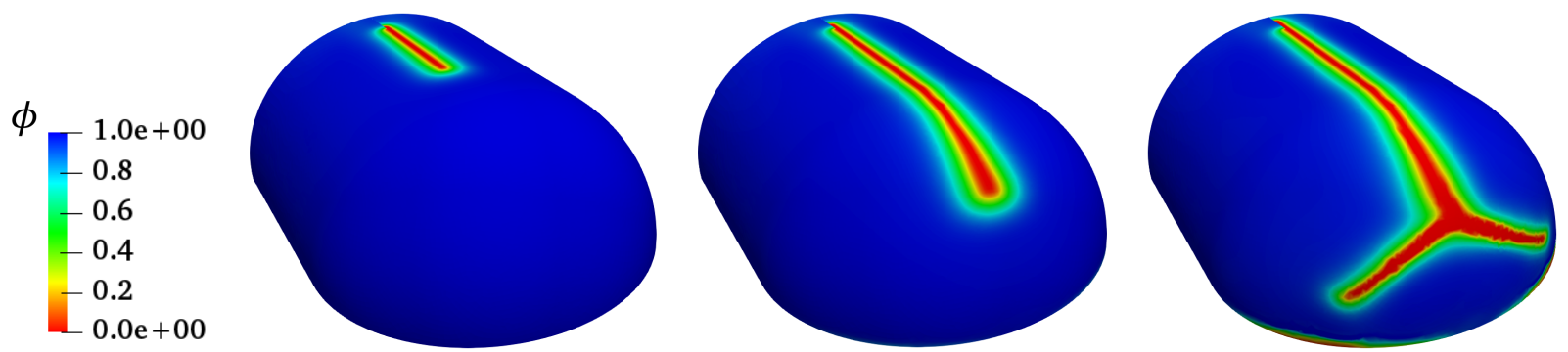

Fig. 24: Notched cylinder with spherical cap under uniform internal pressure: phase field evolution with increasing load-increments $[\phi=1$ and $\phi=0$ represent intact and cracked states of the material $]$

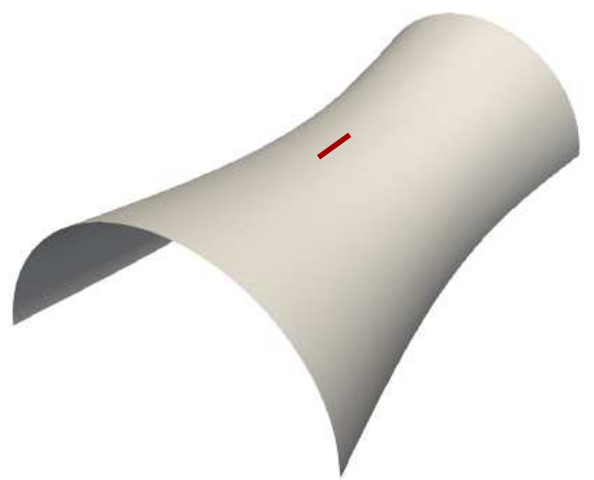

(a)

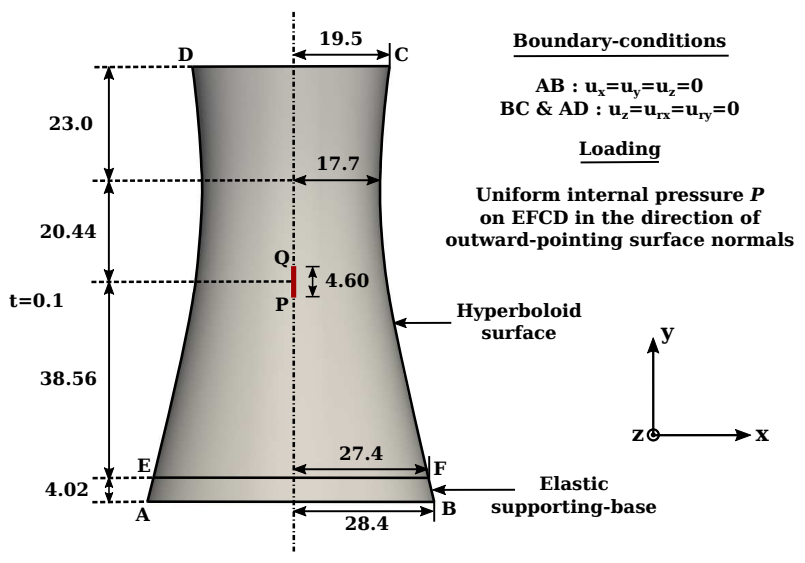

(b)

Fig. 25: Geometry, boundary conditions and loading on the assymetric hyperboloid tower with central notch subjected to uniform internal surface-pressure $P$ (All dimensions in $\mathrm{mm}$ )

tional journal for numerical methods in engineering ${ }_{788}$ 45(5):601-620

789

14. Belytschko T, Leviathan I (1994) Physical stabi-79o lization of the 4-node shell element with one point quadrature. Computer Methods in Applied Me-792 chanics and Engineering 113(3-4):321-350

793

15. Belytschko T, Tsay CS (1983) A stabilization pro-794 cedure for the quadrilateral plate element with one-795 point quadrature. International Journal for Numer-796 ical Methods in Engineering 19(3):405-419

16. Borden MJ, Verhoosel CV, Scott MA, Hughes TJ, Landis CM (2012) A phase-field description of dynamic brittle fracture. Computer Methods in Applied Mechanics and Engineering 217:77-95

17. Borden MJ, Hughes TJ, Landis CM, Anvari A, Lee IJ (2016) A phase-field formulation for fracture in ductile materials: Finite deformation balance law derivation, plastic degradation, and stress triaxial- 

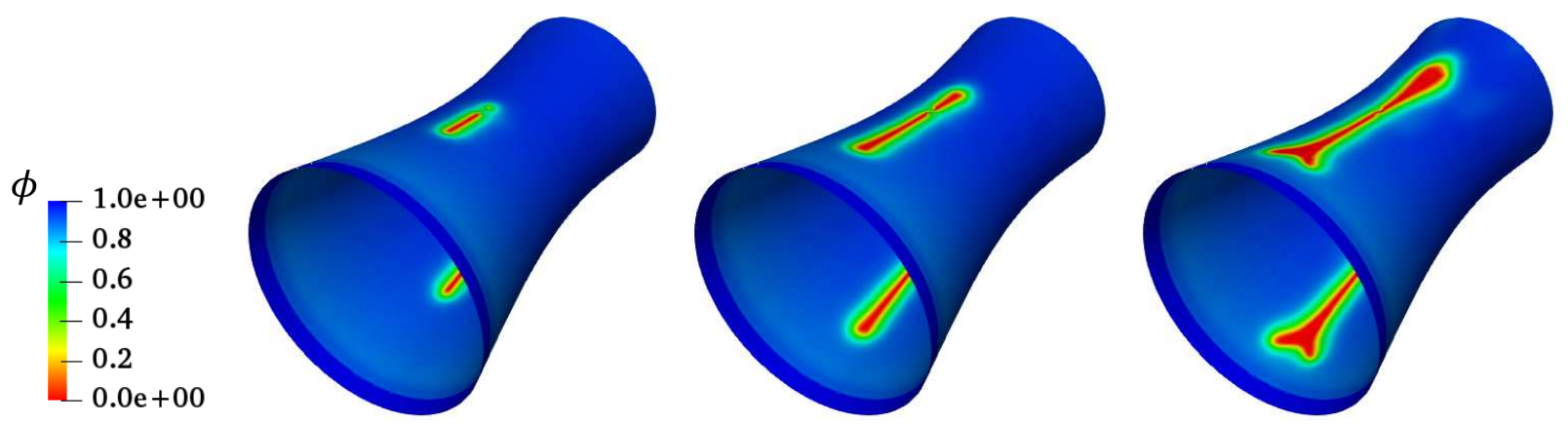

Fig. 26: Assymetric hyperboloid tower under uniform internal pressure: phase field evolution with increasing loadincrements $[\phi=1$ and $\phi=0$ represent intact and cracked states of the material] (Full geometry assembled for better visualization)

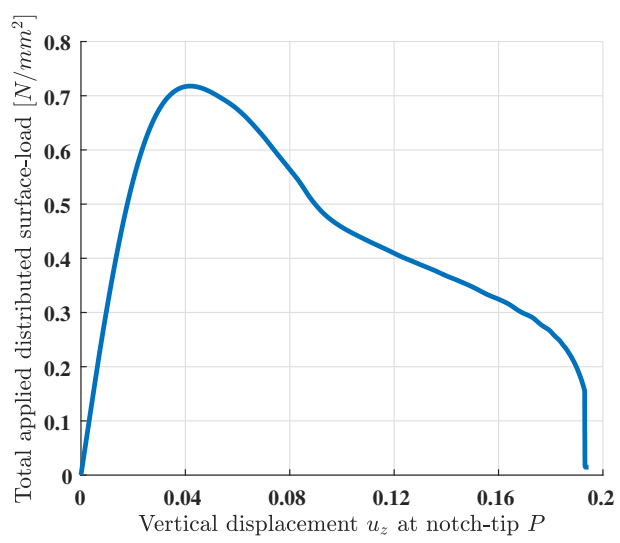

815

816

817

818

819

820

821

822

823

824

Fig. 27: Assymetric hyperboloid tower under uniform 828 internal pressure: Applied pressure load vs vertical dis-829 placement $u_{z}$ measured at the bottom notch-tip $P$
22. Carollo V, Reinoso J, Paggi M (2017) A 3d finite strain model for intralayer and interlayer crack simulation coupling the phase field approach and cohesive zone model. Composite Structures 182:636-651

23. Cook RD, Malkus DS, Plesha ME, Witt RJ (1974) Concepts and applications of finite element analysis, vol 4. Wiley New York

24. Dolbow J, Belytschko T (1999) A finite element method for crack growth without remeshing. International journal for numerical methods in engineering 46(1):131-150

25. Dugdale DS (1960) Yielding of steel sheets containing slits. Journal of the Mechanics and Physics of Solids 8(2):100-104

26. Dvorkin EN, Bathe KJ (1984) A continuum mechanics based four-node shell element for general non-linear analysis. Engineering computations 1(1):77-88

27. Egger A, Pillai U, Agathos K, Kakouris E, Chatzi E, Aschroft IA, Triantafyllou SP (2019) Discrete and phase field methods for linear elastic fracture mechanics: A comparative study and state-of-theart review. Applied Sciences 9(12):2436

28. Ehlers W, Luo C (2018) A phase-field approach embedded in the theory of porous media for the description of dynamic hydraulic fracturing, part II: The crack-opening indicator. Computer Methods in Applied Mechanics and Engineering 341:429-442

29. Francfort GA, Marigo JJ (1998) Revisiting brittle fracture as an energy minimization problem. Journal of the Mechanics and Physics of Solids 46(8):1319-1342

30. Geelen RJ, Liu Y, Hu T, Tupek MR, Dolbow JE (2019) A phase-field formulation for dynamic cohesive fracture. Computer Methods in Applied Mechanics and Engineering 348:680-711 
31. Gerasimov T, De Lorenzis L (2019) On penalizationoo4 in variational phase-field models of brittle fracture.905 Computer Methods in Applied Mechanics and En-906 gineering

32. Griffith AA (1921) The phenomena of rupturesos and flow in solids. Philosophical transactions ofog the Royal Society of London Series A, contain-910 ing papers of a mathematical or physical character 11 221:163-198

912

33. Heider Y, Markert B (2017) A phase-field mod-913 eling approach of hydraulic fracture in saturatedo14 porous media. Mechanics Research Communica-915 tions 80:38-46

916

34. Hillerborg A, Modéer M, Petersson PE (1976) 917 $_{17}$ Analysis of crack formation and crack growth ino18 concrete by means of fracture mechanics and finiter19 elements. Cement and concrete research 6(6):773-920 781

921

35. Ingraffea A, Saouma V (1985) Numerical mod-922 elling of discrete crack propagation in reinforced $d_{23}$ and plain concrete. Fracture Mechanics of concrete 24 pp 171-225

36. Johnson AF, Pickett AK, Rozycki P (2001) Com-926 putational methods for predicting impact damage227 in composite structures. Composites Science and $d_{28}$ Technology 61(15):2183-2192

37. Kakouris E, Triantafyllou S (2018) Material point930 method for crack propagation in anisotropic media:931 a phase field approach. Archive of Applied Mechan-932 ics 88(1-2):287-316

38. Kiendl J, Ambati M, De Lorenzis L, Gomez H, Re-934 ali A (2016) Phase-field description of brittle frac-935 ture in plates and shells. Computer Methods in Ap-936 plied Mechanics and Engineering 312:374-394

39. Ko Y, Lee PS, Bathe KJ (2017) A new MITC4+938 shell element. Computers \& Structures 182:404-418939

40. Kuhn C, Müller R (2010) A continuum phase fieldero model for fracture. Engineering Fracture Mechanics941 $77(18): 3625-3634$

41. Miehe C, Hofacker M, Welschinger F (2010) A A43 phase field model for rate-independent crack prop-944 agation: Robust algorithmic implementation based ${ }^{4} 5$ on operator splits. Computer Methods in Applied946 Mechanics and Engineering 199(45):2765-2778 947

42. Miehe C, Welschinger F, Hofacker M (2010) Ther-948 modynamically consistent phase-field models of 949 fracture: Variational principles and multi-field $\mathrm{FE}_{950}$ implementations. International Journal for Numer-951 ical Methods in Engineering 83(10):1273-1311 952

43. Moës N, Stolz C, Bernard PE, Chevaugeon No53 (2011) A level set based model for damage growth:954 the thick level set approach. International Journalo55 for Numerical Methods in Engineering 86(3):358-956
380

44. Moutsanidis G, Kamensky D, Chen J, Bazilevs Y (2018) Hyperbolic phase field modeling of brittle fracture: Part II - immersed IGA-RKPM coupling for air-blast-structure interaction. Journal of the Mechanics and Physics of Solids 121:114-132

45. Pham K, Marigo JJ (2010) Approche variationnelle de l'endommagement: I. Les concepts fondamentaux. Comptes Rendus Mécanique 338(4):191-198

46. Pham K, Ravi-Chandar K, Landis C (2017) Experimental validation of a phase-field model for fracture. International Journal of Fracture 205(1):83101

47. Pillai U, Heider Y, Markert B (2018) A diffusive dynamic brittle fracture model for heterogeneous solids and porous materials with implementation using a user-element subroutine. Computational Materials Science 153:36-47

48. Quintanas-Corominas A, Turon A, Reinoso J, Casoni E, Paggi M, Mayugo J (2020) A phase field approach enhanced with a cohesive zone model for modeling delamination induced by matrix cracking. Computer Methods in Applied Mechanics and Engineering 358:112618

49. Reinoso J, Arteiro A, Paggi M, Camanho P (2017) Strength prediction of notched thin ply laminates using finite fracture mechanics and the phase field approach. Composites Science and Technology 150:205-216

50. Reinoso J, Paggi M, Linder C (2017) Phase field modeling of brittle fracture for enhanced assumed strain shells at large deformations: formulation and finite element implementation. Computational Mechanics 59(6):981-1001

51. Remmers J, de Borst R, Needleman A (2003) A cohesive segments method for the simulation of crack growth. Computational mechanics 31(1-2):69-77

52. Rethore J, Gravouil A, Combescure A (2004) A stable numerical scheme for the finite element simulation of dynamic crack propagation with remeshing. Computer methods in applied mechanics and engineering 193(42):4493-4510

53. Rouzegar SJ, Mirzaei M (2013) Modeling dynamic fracture in Kirchhoff plates and shells using the extended finite element method. Scientia Iranica 20(1):120-130

54. Scheider I, Brocks W (2006) Cohesive elements for thin-walled structures. Computational Materials Science 37(1-2):101-109

55. Scheider I, Brocks W (2009) Residual strength prediction of a complex structure using crack extension analyses. Engineering Fracture Mechanics 76(1):149-163 
56. Schwab M, Todt M, Wolfahrt M, Pettermann H (2016) Failure mechanism based modelling of impact on fabric reinforced composite laminates based on shell elements. Composites Science and Technology 128:131-137

57. Shahani A, Fasakhodi MA (2009) Finite element analysis of dynamic crack propagation using remeshing technique. Materials \& design ${ }^{996}$ 30(4):1032-1041

58. Sih GC, Paris P, Erdogan F (1962) Crack-tip,998 stress-intensity factors for plane extension and ${ }^{999}$ plate bending problems. Journal of Applied Me $\mathrm{i}^{1000}$ chanics 29(2):306-312

59. Soto A, González E, Maimí P, De La Escalera FM, De Aja JS, Alvarez E (2018) Low velocity impact and compression after impact simulation of thin ply laminates. Composites Part A: Applied Science and Manufacturing 109:413-427

60. Soto A, González E, Maimí P, de la Escalera FM, de Aja JS, Alvarez E (2018) Low velocity impact and compression after impact simulation of thin ply laminates. Composites Part A: Applied Science and Manufacturing 109:413-427

61. Ulmer H, Hofacker M, Miehe C (2012) Phase field modeling of fracture in plates and shells. PAMM 12(1):171-172

62. Wilson ZA, Landis CM (2016) Phase-field modeling of hydraulic fracture. Journal of the Mechanics and Physics of Solids 96:264-290

63. Wu JY, Nguyen VP, Nguyen CT, Sutula D, Bordas S, Sinaie S (2018) Phase field modeling of fracture. Advances in Applied Mechancis: Multi-scale Theory and Computation 52

64. Zienkiewicz O, Taylor R, Too J (1971) Reduced integration technique in general analysis of plates and shells. International Journal for Numerical Methods in Engineering 3(2):275-290

\section{Appendices}

\section{A Jacobian for coordinate transformation}

The Jacobian $[\mathbf{J}]$ for coordinate transformation mapping in a Reissner-Mindlin shell element and its first column are defined as in Eq. (63) and (64). Eq. (64) can be subsequently used to derive expressions for second and third column in a similar manner.

$[J]=\left[\begin{array}{lll}x_{, \xi} & y_{, \xi} & z, \xi \\ x_{, \eta} & y_{, \eta} & z_{, \eta} \\ x_{, \zeta} & y_{, \zeta} & z_{, \zeta}\end{array}\right]$ where,

$\left[\begin{array}{c}x_{, \xi} \\ x_{, \eta} \\ x_{, \zeta}\end{array}\right]=\left[\begin{array}{c}\sum N_{i, \xi}\left(x_{i}+\frac{\zeta t_{i} l_{3 i}}{2}\right) \\ \sum N_{i, \eta}\left(x_{i}+\frac{\zeta t_{i} l_{3 i}}{2}\right) \\ \sum N_{i}\left(\frac{t_{i} l_{3 i}}{2}\right)\end{array}\right]$

where, $\mathbf{x}=[x, y, z]$ is the position vector of any arbitrary point within the shell element, $\{\xi, \eta, \zeta\}$ are the shell parametric coordinates, $t_{i}$ is the shell thickness and $\left\{l_{3 i}, m_{3 i}, n_{3 i}\right\}$ are the direction cosines of normal vector $V_{3 i}$ to the shell mid-surface at any node $i$.

\section{B Coordinate-transformation matrix for rotation of strain tensors}

The strains can be rotated from any one coordinate system (say $C_{1}$ with normalized basis vectors $\bar{e}$ ) to another coordinate system $\left(C_{2}\right.$ with normalized basis vectors $\left.\hat{e}\right)$ by multiplying with the strain-transformation matrix $\mathcal{T}_{\varepsilon}$ shown in eq. (65).

$\mathcal{T}_{\varepsilon}=\left[\begin{array}{ll}\mathcal{T}_{11} & \mathcal{T}_{12} \\ \mathcal{T}_{21} & \mathcal{T}_{22}\end{array}\right]$

with,

$\mathcal{T}_{11}=\left[\begin{array}{lll}l_{1}^{2} & m_{1}^{2} & n_{1}^{2} \\ l_{2}^{2} & m_{2}^{2} & n_{2}^{2} \\ l_{3}^{2} & m_{3}^{2} & n_{3}^{2}\end{array}\right]$

$\mathcal{T}_{12}=\left[\begin{array}{lll}l_{1} m_{1} & m_{1} n_{1} & n_{1} l_{1} \\ l_{2} m_{2} & m_{2} n_{2} & n_{2} l_{2} \\ l_{3} m_{3} & m_{3} n_{3} & n_{3} l_{3}\end{array}\right]$

$\mathcal{T}_{21}=\left[\begin{array}{lll}2 l_{1} l_{2} & 2 m_{1} m_{2} & 2 n_{1} n_{2} \\ 2 l_{2} l_{3} & 2 m_{2} m_{3} & 2 n_{2} n_{3} \\ 2 l_{3} l_{1} & 2 m_{3} m_{1} & 2 n_{3} n_{1}\end{array}\right]$

$\mathcal{T}_{22}=\left[\begin{array}{lll}l_{1} m_{2}+l_{2} m_{1} & m_{1} n_{2}+m_{2} n_{1} & n_{1} l_{2}+n_{2} l_{1} \\ l_{2} m_{3}+l_{3} m_{2} & m_{2} n_{3}+m_{3} n_{2} & n_{2} l_{3}+n_{3} l_{2} \\ l_{3} m_{1}+l_{1} m_{3} & m_{3} n_{1}+m_{1} n_{3} & n_{3} l_{1}+n_{1} l_{3}\end{array}\right]$

where, the terms $\left[l_{1}, m_{1}, n_{1}\right],\left[l_{2}, m_{2}, n_{2}\right]$ and $\left[l_{3}, m_{3}, n_{3}\right]$ correspond to the direction cosines of the shell nodalvectors $V_{1 i}, V_{2 i}$ and $V_{3 i}$ respectively, defined according to Eq. (70) [11].

$l_{1}=\cos \left[\bar{e}_{x}, \hat{e}_{x}\right] ; m_{1}=\cos \left[\bar{e}_{y}, \hat{e}_{x}\right] ; n_{1}=\cos \left[\bar{e}_{z}, \hat{e}_{x}\right]$

$l_{2}=\cos \left[\bar{e}_{x}, \hat{e}_{y}\right] ; m_{2}=\cos \left[\bar{e}_{y}, \hat{e}_{y}\right] ; n_{2}=\cos \left[\bar{e}_{z}, \hat{e}_{y}\right]$

$l_{3}=\cos \left[\bar{e}_{x}, \hat{e}_{z}\right] ; m_{3}=\cos \left[\bar{e}_{y}, \hat{e}_{z}\right] ; n_{3}=\cos \left[\bar{e}_{z}, \hat{e}_{z}\right]$

The resulting $\mathcal{T}_{\varepsilon}$ is a $(6 \times 6)$ matrix which can be multiplied to $(6 \times 1)$ strain vector (expressed in Voigt notation) to transform it from coordinate system $C_{1}$ to coordinate system $C_{2}$. 